EConomía Teoría y Práctica Nueva Época, número 48, enero-junio 2018, pp. 75-124,

http://dx.doi.org/10.24275/ETYPUAM/NE/482018/Perez

\title{
Determinantes de la capacidad tecnológica en América Latina: una aplicación empírica con datos de panel*
}

\section{Determinants of Technological Capacity in Latin America: An Empirical Application with Panel Data}

\author{
Carla Carolina Pérez Hernández**, Denise Gómez Hernández*** \\ y Graciela Lara Gómez****
}

\begin{abstract}
RESUMEN
El objetivo del presente trabajo es examinar los determinantes de la capacidad tecnológica en Latinoamérica. Para ello se desarrolló un modelo econométrico utilizando la técnica de datos de panel con efectos fijos, con base en el set de indicadores propuesto por Comisión Económica para América Latina y el Caribe (CEPAL, 2007) y Chinaprayoon (2007), y recopilando los datos de diversas fuentes públicas, utilizando una serie temporal de 14 años y una dimensión transversal de 17 países; esto con el fin de analizar el grado de impacto de las variables relativas a la capacidad tecnológica en América Latina. A partir de la estimación econométrica, se encuentra que la apertura comercial, el stock de patentes y el Gasto en I+D, son los determinantes más influyentes de la capacidad tecnológica en dicho contexto. Finalmente, se realiza la triangulación de los hallazgos empíricos con las implicaciones en materia de Ciencia, Tecnología e Innovación (СТI), a fin de generar discusiones y reflexiones proclives a incidir en mejores políticas en materia tecnocientífica para la región.
\end{abstract}

Palabras clave: Capacidad tecnológica, panel de datos, efectos fijos, Latinoamérica.

Códigos JEL: O10, O30, O31

\begin{abstract}
The aim of this paper is to evaluate the determinants of technological capacity in Latin America. We developed an econometric model using the technique of panel data with fixed effects, based on the indicators proposed by CEPAL (2007) \& Chinaprayoon (2007). Then, we compiled data from various public sources, with a time series of 14 years and a transversal dimension of 17 countries; in order to analyze the impact of variables related to the technological capacity in Latin America. The results show that the level of trade liberalization, the patent stock and the expenditure on $R \& D$ are the most influential determinants of technological capacity in this context. Finally, we reflected the best practices of Science, Technology and Innovation, in order to generate discussions to influence policies in techno-scientific issues for the region.
\end{abstract}

Keywords: Technological capabilities, panel data, fixed effects, Latin America.

JEL codes: O10, O30, $\mathrm{O} 31$

*Fecha de recepción: 23-06-2016. Fecha de aprobación: 13-12-2017.

** Universidad Autónoma de Querétaro. Correo: carolina.cph@gmail.com. ORCID: 00000001-8286-8775

*** Universidad Autónoma de Querétaro. Correo: actdenise@gmail.com. ORCID: 0000-0001$5562-9541$

**** Universidad Autónoma de Querétaro. Correo: glaragomez@yahoo.com.mx. ORCID: 0000-0001-9984-7372 


\section{INTRODUCCIÓN}

Es bien sabido que el patrón de comportamiento de la capacidad tecnológica en países desarrollados difiere de los países en vías de desarrollo. Sin embargo, la mayoría de los estudios empíricos analizan a profundidad a los primeros, en parte porque las economías desarrolladas reconocen a la ciencia, tecnología e innovación como piezas fundamentales para sus estrategias de crecimiento sostenido, pero también en parte por el amplio acervo estadístico con el que cuentan estos países. Por el contrario, los países en vías de desarrollo no cuentan con el mismo repertorio de estudios, aun a pesar de que el tema de la capacidad tecnológica mantiene la misma importancia estratégica bajo cualquier contexto. Dado tal escenario, se encuentra en Chinaprayoon (2007) un estudio pionero dirigido a modelar la capacidad tecnológica en regiones en desarrollo, bajo el lema "los países desarrollados son animales diferentes a los que están en desarrollo"; sin embargo, el alcance de su estudio en países latinoamericanos es limitado, en parte por la carencia de datos estadísticos disponibles al año 2003 y en parte porque esta región no es foco de su análisis. Bajo el panorama anterior, se observa pertinente enfocar esta clase de investigaciones a regiones en vías de desarrollo y, por primera vez, acogiendo una muestra representativa de países de América Latina, sabiendo que dichos estudios tienen como fin proveer evidencia empírica sobre cuáles son los factores determinantes de la capacidad tecnológica e identificar en qué medida la impactan. Además, mediante ellos se puede fundamentar una mejor política tecnológica, ya que según algunos autores (Ulku, 2004; Schumpeter, 2005; Brunner, 2011; Dosi, 2008; Diaconu, 2011), el éxito de cualquier economía requiere de un alto grado de innovación y por tanto, los países necesitan prioritariamente medir su capacidad tecnológica. En ese sentido, Stern, Porter, y Furman (2000) apuntan que "la evaluación de los determinantes de la capacidad tecnológica e innovativa en todos los países tiene un doble objetivo: primero el de informar a los encargados de la política de ciencia y tecnología y un segundo propósito es el de iluminar los factores que subyacen en el crecimiento de la productividad nacional".

Por otra parte, la importancia del tema es que, a nivel macroeconómico, las capacidades tecnológicas son consideradas las habilidades más amplias que se requieren para iniciar un proceso de mejoras conducentes a un sendero de crecimiento y desarrollo sostenido. Estas capacidades, por tanto, deben complementarse de manera tal que se produzca una nueva combinación de ideas existentes, capacidades, habilidades, recursos, entre otros factores. El resultado de esta nueva combinación puesta en el mercado es lo que se conoce como innovación, por consecuencia, el estudio de dicho tema a nivel macro es una de las principales preocupaciones de la literatura sobre los determinantes del crecimiento y el 
desarrollo económico (CEPAL, 2007). Además, Chinaprayoon (2007), señala que indiscutiblemente, los países en desarrollo necesitan contar con la medición periódica de sus capacidades de CTI con el fin de poder adaptar dichas políticas para ayudar a mejorar sus capacidades tecnológicas y por consecuencia sus niveles de innovación.

El artículo se desarrolla contemplando los siguientes apartados: $a$ ) revisión de la literatura (estudios empíricos previos) relacionados con la capacidad tecnológica; $b$ ) metodología de investigación; $c$ ) los resultados descriptivos y econométricos; d) conclusiones y discusiones.

\section{BREVE REVISIÓN DE LA LITERATURA (ESTUdIOS PREVIOS)}

Bell, Pavitt y Lall (citado por CEPAL, 2007) señalan que el desarrollo de capacidades tecnológicas "implica conocimientos y habilidades para adquirir, usar, absorber, adaptar, mejorar y generar nuevas tecnologías". Partiendo de esta definición se entiende que las capacidades tecnológicas incluyen las capacidades de innovación y las de absorción. Las primeras están sujetas a aspectos como las infraestructuras, las actividades propias de innovación, formación de capital humano, y las habilidades de los países para crear, imitar y gestionar el conocimiento, mientras que la segunda refiere a la posibilidad de acceder, aprender y asimilar tecnologías extranjeras (Quiñones y Tezanos, 2011). Consecuentemente, la conexión existente entre dichas capacidades, hace pertinente la focalización hacia las capacidades tecnológicas como elemento central que antecede a la innovación tecnológica.

En ese sentido, hallamos en la literatura que el pensamiento evolucionista enfatiza que el desarrollo económico depende fundamentalmente de la innovación y de la capacidad tecnológica que le subyace, propiamente la Teoría Económica Endógena $^{1}$ y la Teoría Económica Evolutiva o corriente neoschumpeteriana ${ }^{2}$, tienen como argumento principal que una mayor creación y acumulación de capacidades tecnológicas, conllevará a mayores niveles de innovación y esto a su vez, tendrá repercusiones positivas en el desarrollo económico en sus tres dimensiones: económica, social y ambiental.

${ }^{1}$ Las teorías modernas del desarrollo económico endógeno, al sustituir la tendencia de la inversión en capital por la inversión en el conocimiento, ponen a la ciencia y a la tecnología en el primer plano de importancia para la competitividad en un mundo globalizado (Osorio, 2009).

${ }^{2}$ La Teoría Económica Evolucionista (TEE), "llamada también corriente neoschumpeteriana, que comparte el postulado fundamental propuesto por Joseph Schumpeter acerca del capitalismo como un sistema económico caracterizado por la emergencia de innovaciones técnicas y organizativas endógenas que dan lugar a la existencia de ciclos del desarrollo del sistema”, (Borrastero, 2012). 
Teorías recientes del crecimiento económico ponen atención al cambio tecnológico endógeno para explicar los patrones de crecimiento de las economías mundiales (Ulku, 2004). Paralelamente, se identifica que (Stern, Porter, y Furman, 2000; Baumert, Thomas, Heijs y Joost, 2002; Ulku, 2004; Chinaprayoon, 2007; Hu y Mathews, 2008; Gans y Hayes, 2009) los modelos econométricos construidos están principalmente basados en el modelo de crecimiento endógeno propuesto por Romer (1990), en donde la innovación o el flujo de nuevas ideas $(\AA)$ está en función del capital humano en los sectores de desarrollo tecnológico $\left(\mathrm{H}_{\mathrm{A}}\right)$ y el stock de conocimiento en la economía (A). Esta relación está indicada por la siguiente función de producción basada en el conocimiento:

$$
\AA=\delta H_{A}^{\theta}{ }_{A}
$$

De acuerdo con Chinaprayoon (2007), en el modelo de Romer, la producción de innovación es lineal tanto en el capital humano dentro de los sectores de I+D como en el stock de conocimiento; y por lo tanto a largo plazo,

$\theta=0$. Además se denota que este modelo, sucesivamente, lleva a una economía de desarrollo sustentable por dos razones:

1) a mayor capital humano dedicado a los sectores de desarrollo tecnológico, mayor innovación será generada.

2) entre más grande sea el stock inicial de conocimiento que posea una economía, más alto será el nivel productividad que ésta pueda alcanzar.

En consecuencia, Ulku (2004) distingue que la ecuación de regresión para investigar los determinantes de la capacidad tecnológica, se deriva de la ecuación (1) y da pie a la ecuación (2) en donde, Å es el flujo de la capacidad tecnológica (o flujos de conocimiento, como en el modelo de Romer, A es el stock de la innovación (o el conocimiento de valores) y $\mathrm{H}$ es el capital humano dedicado a I + D. Tal como se muestra a continuación:

$$
\AA=A H^{\theta}
$$

Derivado de lo anterior, (Stern, Porter, y Furman, 2000; Ulku, 2004; Chinaprayoon, 2007), realizan una serie de estudios econométricos y encuentran que el modelo de Romer puede caracterizar la capacidad tecnológica de un país para innovar, dada la función de producción del desarrollo tecnológico (I+D), tal como se muestra en la ecuación (3), la cual puede ser representada en forma logarítmica: 
$\log (\AA)=\log (\mathrm{A})+\theta \log (\mathrm{H})$

La ecuación (3) implica que un crecimiento en 1 por ciento de $\mathrm{A}$ y $\mathrm{H}$, se incrementa la capacidad tecnológica $(\AA)$, por 1 por ciento y $\theta$ por ciento respectivamente. En el modelo de Romer, se asume que $\theta$ es igual a uno. ${ }^{3}$

Chinaprayoon (2007) genera por primera vez un modelo econométrico (basado en Romer) para identificar los determinantes de la capacidad tecnológica específicamente de países en desarrollo (los estudios previos, exploraban esta capacidad exclusivamente en países desarrollados altamente industrializados). Sin embargo, el alcance de su estudio en países latinoamericanos es limitado, ${ }^{4}$ principalmente porque esta región no está en su perímetro de estudio. Propiamente, la fórmula utilizada por este autor, contempla que los outputs de la innovación (capacidad tecnológica) están en función de los inputs de I+D, los factores habilitadores y las variables de control. Como factores de salida (outputs), ocupa por primera vez, variables propias de países en desarrollo, específicamente, adopta estadísticas de patentes y exportaciones de alta tecnología.

$$
\text { Outputs }=f(\text { Variables de } \mathrm{I}+\mathrm{D} \text {, factores habilitadores })
$$

Una característica común de los países en desarrollo es que la capacidad tecnológica local en ellos es generalmente baja. Por lo tanto, se vuelve indispensable tomar en cuenta el rol de la fuerza global y especialmente las importaciones/exportaciones de tecnología.

Además Chinaprayoon (2007), considera necesario que los países en desarrollo cuenten con una batería propia de indicadores dado que la estructura de ciencia y tecnología en dichos lugares difiere de los países desarrollados (los cuales han sido ampliamente estudiados, mientras que el estudio de los primeros, es muy incipiente). Análogamente, CEPAL (2007), realiza un estudio estadístico descriptivo de la capacidad tecnológica en América Latina, planteando un set de medición de las capacidades tecnológicas para Países en Desarrollo (PED) mediante tres dimensiones: 1) la base disponible (recursos humanos, infraestructura, 'calidad' del entorno), 2) los esfuerzos realizados para el incremento y consolidación de las capacidades (adquisición de conocimiento en sus diversas formas, I+D, y otras) y 3) los

${ }^{3}$ Revisar Romer (1990).

${ }^{4}$ Los países latinoamericanos considerados en desarrollo por el Banco Mundial (2015) son 28, de los cuales Chinaprayoon (2007), en su estudio empírico únicamente considera 10 en un periodo de 1996-2003. 
resultados logrados a partir de las capacidades existentes (patentes, tasa de innovación y contenido tecnológico de las exportaciones). Para CEPAL (2007), la capacidad tecnológica entonces, se identifica mediante la siguiente ecuación:

\section{Los resultados logrados $=f$ (La base disponible, los esfuerzos realizados)}

Se percibe que tanto Chinaprayoon (2007) como CEPAL (2007), al enfocarse en la medición de capacidades tecnológicas en países en desarrollo, adoptan un set de indicadores muy similar (véase cuadro 1) en el que convergen variables contextuales (base disponible/factores habilitadores) y variables de entrada (esfuerzos realizados/variables de I+D).

Cuadro 1. Variables del estudio

\begin{tabular}{|c|c|c|}
\hline \multicolumn{2}{|l|}{ VARIABLES INDEPENDIENTES $(X)$} & VARIABLES DEPENDIENTES (Y) \\
\hline Base Disponible (Enabling factors) & $\begin{array}{l}\text { Esfuerzos realizados ( } R \text { \& D } \\
\text { inputs) }\end{array}$ & Resultados logrados (outputs) \\
\hline $\begin{array}{l}\text { - Tasa de alfabetización } \\
\text { - Enrolamiento (primario, secundario y terciario) } \\
\text { - Graduados en ciencia y tecnología } \\
\text { - Personas dedicadas a I+D } \\
\text { - Internet } \\
\text { - Líneas telefónicas } \\
\text { - Consumo de energía per cápita (kWh per cápita) } \\
\text { - Complejidad de la demanda tecnológica } \\
\text { - Producto Interno Bruto per cápita } \\
\text { - Apertura (exportaciones + importaciones / PIB) } \\
\text { - Stock de patentes }\end{array}$ & $\begin{array}{l}\text { - Gasto en educación públi- } \\
\text { ca (\% of PIB) } \\
\text { - Gasto en Investigación y } \\
\text { Desarrollo (\% PIB) } \\
\text { - Adquisición externa de co- } \\
\text { nocimiento: Pagos de licen- } \\
\text { cias } \\
\text { - Inversión Extranjera Directa } \\
\text { (IED,\% PIB) }\end{array}$ & $\begin{array}{l}\text { - Patentes otorgadas } \\
\text { - Solicitudes de patentes } \\
\text { - Publicaciones científicas } \\
\text { - Exportaciones de contenido tec- } \\
\text { nológico } \\
\text { VARIABLES DE CONTROL (Y) } \\
\text { - PIB per cápita } \\
\text { - Productividad Total de los Facto- } \\
\text { res }\end{array}$ \\
\hline
\end{tabular}

Fuente: Elaboración propia con base en CEPAL (2007) y Chinaprayoon (2007).

En suma, la revisión de estudios empíricos al respecto, nos muestra un amplio repertorio de estudios econométricos en países desarrollados, mientras que en países en vías de desarrollo, los análisis son incipientes y requieren de estudios más amplios o complementarios. Específicamente en América Latina, se encuentra un trabajo parteaguas en CEPAL (2007), sin embargo, tal como lo señalan en sus limitantes de estudio, a la fecha de ejecución de dicha investigación, las carencias de información condicionaron severamente las posibilidades del análisis, pudiendo solamente realizar un análisis descriptivo de la condición Latinoamericana de capacidades tecnológicas.

En el siguiente cuadro, se pueden apreciar los principales estudios empíricos previos relacionados principalmente con la modelación econométrica y análisis estadísticos de la capacidad tecnológica y de innovación junto con la metodología utilizada, los indicadores y las aportaciones de dichas investigaciones. 
Cuadro 2. Estudios empíricos previos

\begin{tabular}{|c|c|c|c|}
\hline Autor & Metodología & Indicadores/Muestra & Aportaciones \\
\hline Romer (1990) & $\begin{array}{l}\text { Modelo matemá- } \\
\text { tico }\end{array}$ & NA & $\begin{array}{l}\text { El crecimiento económico es endógeno, porque depende de la } \\
\text { respuesta de los agentes a los incentivos de mercado en la pro- } \\
\text { ducción más la optimización del consumidor. } \\
\text { La característica fundamental del modelo de Romer es que el } \\
\text { conocimiento entra en la producción de dos formas distintas: 1) } \\
\text { Un nuevo diseño permite que la producción de nuevo bien de } \\
\text { capital se puede utilizar para producir una salida. } 2 \text { ) Un nuevo } \\
\text { diseño también aumenta el acervo total de conocimientos lo que } \\
\text { aumenta la productividad del capital humano en el sector de la } \\
\text { investigación. }\end{array}$ \\
\hline $\begin{array}{l}\text { Stern, Porter, \& } \\
\text { Furman (2000; } \\
\text { 2002) }\end{array}$ & $\begin{array}{l}\text { Modelo eco- } \\
\text { nométrico con } \\
\text { efectos fijos }\end{array}$ & $\begin{array}{l}12 \text { indicadores } \\
17 \text { países de la oCDE. Ningún } \\
\text { país latinoamericano } \\
\text { Periodo: } 1973-1996\end{array}$ & $\begin{array}{l}\text { Los resultados sugieren que la política pública desempeña un } \\
\text { papel importante en la formación de la capacidad tecnológica } \\
\text { nacional de un país. Más allá de simplemente aumentar el nivel } \\
\text { de recursos de I + D a disposición de la economía, la agenda } \\
\text { política del gobierno juega un papel importante en la formación } \\
\text { de capital de inversión e innovación incentivos humanos. Cada } \\
\text { uno de los países que han aumentado su nivel estimado de la } \\
\text { capacidad innovadora en el último cuarto de siglo, han puesto en } \\
\text { práctica políticas que estimulan la inversión de capital humano } \\
\text { en ciencia e ingeniería, así como la competencia sobre la base de } \\
\text { la innovación (por ejemplo, mediante la aprobación de créditos } \\
\text { fiscales a la I + D y la apertura gradual de los mercados a la } \\
\text { competencia internacional). }\end{array}$ \\
\hline Ulku (2004) & $\begin{array}{l}\text { Modelo eco- } \\
\text { nométrico con } \\
\text { efectos fijos y } \\
\text { aleatorios }\end{array}$ & $\begin{array}{l}10 \text { indicadores } \\
30 \text { países desarrollados y } 3 \\
\text { latinoamericanos } \\
\text { Periodo: } 1981-1997\end{array}$ & $\begin{array}{l}\text { Los resultados sugieren una relación positiva entre el PIB per cá- } \\
\text { pita y la innovación, tanto en la OCDE y los países fuera de la OCDE, } \\
\text { mientras que el efecto del stock de I + D en la innovación es } \\
\text { significativa sólo en los países de la oCDE con grandes mercados. } \\
\text { Aunque estos resultados proporcionan apoyo a modelos de cre- } \\
\text { cimiento endógeno, no hay evidencia de rendimientos constantes } \\
\text { a la innovación en términos de I + D, lo que implica que la inno- } \\
\text { vación no conduce a aumentos permanentes en el crecimiento } \\
\text { económico. Sin embargo, estos resultados no responden necesa- } \\
\text { riamente a un rechazo de los modelos de crecimiento basados en } \\
\text { I + D, dado que ni la patente ni datos sobre I + D reflejan la to- } \\
\text { talidad de las actividades de I + D y la innovación. }\end{array}$ \\
\hline $\begin{array}{l}\text { Gans \& Hayes } \\
(2009)\end{array}$ & $\begin{array}{l}\text { Modelo eco- } \\
\text { nométrico con } \\
\text { efectos fijos }\end{array}$ & $\begin{array}{l}10 \text { indicadores } \\
29 \text { países de la oCDE. } \\
1 \text { país latinoamericano } \\
\text { Periodo: } 1973-2008\end{array}$ & $\begin{array}{l}\text { En una economía global, la competitividad basada en la innova- } \\
\text { ción proporciona una base más estable para el crecimiento de la } \\
\text { productividad que el énfasis tradicional en la producción de bajo } \\
\text { costo. Australia debe construir sobre los cimientos de la apertura } \\
\text { a la competencia internacional y la protección de la propiedad } \\
\text { intelectual. Sin embargo, Australia tiene que centrarse en las } \\
\text { áreas que parecen haberse descuidado en las últimas dos déca- } \\
\text { das. En particular, debería asegurar una piscina de clase mundial } \\
\text { de innovadores formados mediante el mantenimiento de un alto } \\
\text { nivel de excelencia universitaria con estímulos a los estudiantes } \\
\text { a seguir la ciencia y carreras de ingeniería. }\end{array}$ \\
\hline
\end{tabular}


82 ECONOMÍA TeOría Y PRÁCTICA • Nueva Época, número 48, enero-junio 2018

\begin{tabular}{|c|c|c|c|}
\hline Autor & Metodología & Indicadores/Muestra & Aportaciones \\
\hline $\begin{array}{l}\text { Baumert, Tho- } \\
\text { mas, Heijs \& } \\
\text { Joost (2002) }\end{array}$ & $\begin{array}{l}\text { Modelo economé- } \\
\text { trico MCO }\end{array}$ & $\begin{array}{l}11 \text { indicadores } \\
15 \text { Comunidades Autónomas } \\
\text { Españolas } \\
\text { Periodo: } 1995-1997\end{array}$ & $\begin{array}{l}\text { Una de las principales hipótesis a comprobar para el caso español, } \\
\text { era la incidencia que el esfuerzo tecnológico llevado a cabo por las } \\
\text { regiones así como el tamaño tecnológico-empresarial de las mis- } \\
\text { mas ejerce sobre el resultado innovador regional. Tal y como cabía } \\
\text { esperar, se ha podido constatar el impacto positivo que sobre el } \\
\text { output tecnológico tiene el esfuerzo innovador, independiente- } \\
\text { mente de que sea medido a través del stock de capital tecnológico, } \\
\text { del gasto en I+D o del personal total en I+D. }\end{array}$ \\
\hline $\begin{array}{l}\text { Chinaprayoon } \\
\text { (2007) }\end{array}$ & $\begin{array}{l}\text { Modelo economé- } \\
\text { trico con efectos } \\
\text { fijos }\end{array}$ & $\begin{array}{l}15 \text { indicadores } \\
38 \text { países en desarrollo } \\
\text { de los cuales } 10 \text { son latinoa- } \\
\text { mericanos } \\
\text { Periodo: } 1996-2003\end{array}$ & $\begin{array}{l}\text { A partir de la estimación econométrica, se encontró que las con- } \\
\text { tribuciones de I+D, la importación de tecnología y la conectividad } \\
\text { internacional son determinantes influyentes de la capacidad tec- } \\
\text { nológica en la muestra de países en desarrollo. A partir de este } \\
\text { descubrimiento, se desarrolló el Índice de Capacidad de Innova- } \\
\text { ción pronosticada para países en desarrollo (INNÔDEX), un indicador } \\
\text { compuesto que clasifica a los países según sus capacidades inno- } \\
\text { vadoras }\end{array}$ \\
\hline CEPAL (2007) & $\begin{array}{l}\text { Análisis estadísti- } \\
\text { co descriptivo }\end{array}$ & $\begin{array}{l}22 \text { indicadores } \\
18 \text { países Latinoaméricanos } \\
\text { Periodo: } 1990-2004\end{array}$ & $\begin{array}{l}\text { En conclusión, todo indica que, a fin de lograr mejores resultados } \\
\text { en materia de cambio tecnológico y un achicamiento de la brecha } \\
\text { con respecto a las naciones más desarrolladas, estos países debe- } \\
\text { rán aumentar significativamente los esfuerzos destinados a una } \\
\text { sustancial mejora de la base disponible, sobre todo en lo que } \\
\text { respecta a un salto significativo en las capacidades de absorción } \\
\text { y una mayor y más equilibrada disponibilidad de infraestructura. }\end{array}$ \\
\hline $\begin{array}{l}\text { García, Bláz- } \\
\text { quez \& López } \\
\text { (2012) }\end{array}$ & $\begin{array}{l}\text { Análisis estadísti- } \\
\text { co multivariante } \\
\text { de cluster }\end{array}$ & $\begin{array}{l}13 \text { indicadores } \\
18 \text { países Latinoaméricanos y } \\
\text { España } \\
2002 \text { y } 2009\end{array}$ & $\begin{array}{l}\text { Los resultados muestran la existencia de cuatro grupos de países } \\
\text { que se definen por una distinta capacidad de innovación tecnoló- } \\
\text { gica, tanto en lo referente a política tecnológica gubernamental y } \\
\text { empresarial como en lo relativo a infraestructuras tecnológicas y } \\
\text { capital humano. Los grupos también difieren en su evolución en } \\
\text { relación con estos factores en el periodo considerado. }\end{array}$ \\
\hline
\end{tabular}

Fuente: Elaboración propia con base en (Stern, Porter, y Furman, 2000; Baumert, Thomas, Heijs y Joost, 2002; Ulku, 2004; Chinaprayoon, 2007; Hu y Mathews, 2008; Gans y Hayes, 2009; CEPAL, 2007; García, Blázquez y López (2012).

A pesar de que estas perspectivas comparten elementos comunes, cada uno enfatiza en diferentes series de tiempo, perímetros de estudio y variables relacionadas con la producción nacional de innovación. Sin embargo, siguiendo a CEPAL (2007), se introduce para efectos de esta investigación el concepto de capacidad tecnológica para integrar las perspectivas anteriores y proporcionar un marco para la prueba empírica entre países latinoamericanos.

\section{Metodología}

La metodología del presente trabajo, consiste en desarrollar un análisis econométrico para identificar los determinantes de la capacidad tecnológica en Latinoamérica (17 países) con una serie de tiempo que va del año 2000 al 2014. Dicho análisis se completa a través de seis etapas: $a$ ) Presentar la estadística descriptiva de las 
variables previamente normalizadas por el tamaño de la población de cada economía. b) Elaborar la matriz de correlaciones de las variables independientes para evitar problemas de multicolinealidad. c) Transformar las variables independientes resultantes y las variables dependientes predeterminadas en Logaritmo Natural (Ln) -con excepción de las que estén expresadas en forma porcentual-. d) Definir las ecuaciones de regresión conforme la ecuación (3): $\log (\AA)=\log (A)+\theta \log (H)$. e) Correr las regresiones en base a la técnica de estimación de panel de datos con efectos fijos. Y finalmente, $f$ ) Interpretar los resultados obtenidos. Las fases mencionadas se describirán puntualmente en las siguientes secciones.

\section{II.1. Variables del estudio}

Todas las variables se enlistan en el cuadro 3 y estarán normalizadas por las series de población para poder tomar en cuenta el tamaño de la economía de cada país. Adicionalmente como se sugiere por la mayoría de los estudios previos (Stern, Porter, y Furman, 2000; Ulku, 2004; Chinaprayoon, 2007; Hu y Mathews, 2008; Gans y Hayes, 2009), los modelos toman la forma funcional Log-Log para lograr minimizar los problemas de valores atípicos. Consecuentemente, todas las variables serán recalculadas en valores de Logaritmo Natural (Ln) para la ecuación (3), excepto por aquellas variables que están ya expresadas en valores porcentuales. Los detalles y definiciones de las variables se muestran a continuación.

Cuadro 3. Variables y definiciones

\begin{tabular}{|c|c|c|c|}
\hline Variables & Nombres & Definiciones & Fuentes \\
\hline \multicolumn{4}{|c|}{ VARABLES DEPENDIENTES (Y) } \\
\hline \multicolumn{4}{|c|}{ Resultados logrados } \\
\hline PATENTES j,t & $\begin{array}{l}\text { Patentes internacionales otor- } \\
\text { gadas por millón de habitantes }\end{array}$ & $\begin{array}{l}\text { Número de patentes otorgadas por } \\
\text { uspTo por millón de habitantes al } \\
\text { año de otorgamiento. }\end{array}$ & UsPto Base de Datos de Patentes \\
\hline PATENTES_APLI j,t & $\begin{array}{l}\text { Las solicitudes de patentes in- } \\
\text { ternacionales por millón de ha- } \\
\text { bitantes }\end{array}$ & $\begin{array}{l}\text { Número de solicitudes de patentes } \\
\text { por uspTo por millón de habitantes }\end{array}$ & uspto Base de Datos de Patentes \\
\hline PUBLICACION j,t & $\begin{array}{l}\text { Publicaciones científicas por mi- } \\
\text { llón de habitantes }\end{array}$ & $\begin{array}{l}\text { Número de artículos científicos y } \\
\text { técnicos de revistas por millón ha- } \\
\text { bitantes en el año de publicación }\end{array}$ & Banco Mundial (wol) \\
\hline COBROLICENCIA j,t & $\begin{array}{l}\text { Recibos de regalías y licencias } \\
\text { por millón de habitantes }\end{array}$ & $\begin{array}{l}\text { Recibos de regalías y licencias de } \\
\text { los recibos por millón de habitan- } \\
\text { tes en el año de cobro (PPA) }\end{array}$ & Banco Mundial (wDI) \\
\hline EXPORTACIONESHT j,t & $\begin{array}{l}\text { Exportación de Alta Tecnología } \\
\text { por millón de habitantes }\end{array}$ & $\begin{array}{l}\text { Valor de las exportaciones de alta } \\
\text { tecnología por millón de habitan- } \\
\text { tes en el año de exportación (PPA) }\end{array}$ & Banco Mundial (wol) \\
\hline
\end{tabular}


84 ECONOMÍA TeORÍA Y PRÁCTICA • Nueva Época, número 48, enero-junio 2018

\section{Cuadro 3. continuación}

\begin{tabular}{|c|c|c|c|}
\hline \multicolumn{4}{|c|}{ VARIABLES INDEPENDIENTES (X) } \\
\hline \multicolumn{4}{|c|}{ Base Disponible } \\
\hline STOCKPATENTj,t & $\begin{array}{l}\text { Stock de patentes internaciona- } \\
\text { les por millones de habitantes }\end{array}$ & $\begin{array}{l}\text { Patentes otorgadas (número acu- } \\
\text { mulado) por uspTo por millón de } \\
\text { habitantes de } 1970 \text { al } 2014\end{array}$ & uspto Base de Datos de Patentes \\
\hline ELECTRICIDADj,t & $\begin{array}{l}\text { Consumo eléctrico por mil habi- } \\
\text { tantes }\end{array}$ & $\begin{array}{l}\text { El consumo de electricidad por ca- } \\
\text { da mil habitantes (Kwah) }\end{array}$ & Banco Mundial (wol) \\
\hline TELj,t & $\begin{array}{l}\text { líneas principales de teléfono } \\
\text { por cada mil habitantes }\end{array}$ & $\begin{array}{l}\text { Número de líneas telefónicas por } \\
\text { cada mil habitantes }\end{array}$ & Banco Mundial (wDI) \\
\hline CELj,t & $\begin{array}{l}\text { Usuarios de teléfonos móviles } \\
\text { por cada mil habitantes }\end{array}$ & $\begin{array}{l}\text { Número de usuario del teléfono } \\
\text { móvil por cada mil habitantes }\end{array}$ & Banco Mundial (wol) \\
\hline INTERNETj,t & $\begin{array}{l}\text { Usuarios de Internet por cada } \\
\text { mil habitantes }\end{array}$ & $\begin{array}{l}\text { Número de usuarios de Internet } \\
\text { por cada mil habitantes }\end{array}$ & Banco Mundial (wDI) \\
\hline GIDE\%PIBj,t & $\begin{array}{l}\text { Gasto total en I + D como por- } \\
\text { centaje del PIB }\end{array}$ & $\begin{array}{l}\text { Gasto total en I + D (PPA) como } \\
\text { porcentaje del PIB (PPA) }\end{array}$ & $\begin{array}{l}\text { Instituto de Estadística de unESCO, } \\
\text { RICYT, oCDE principales Indicadores de } \\
\text { Ciencia y Tecnología, Oficinas Nacio- } \\
\text { nales de Estadística }\end{array}$ \\
\hline GIDEj,t & Gasto per capita I+D & Gasto per capita I+D (PPA) & $\begin{array}{l}\text { Instituto de Estadística de UNESCO, } \\
\text { RICYT, OCDE principales Indicadores de } \\
\text { Ciencia y Tecnología, Oficinas Nacio- } \\
\text { nales de Estadística }\end{array}$ \\
\hline I+DPERSONj,t & $\begin{array}{l}\text { Personal de I + D de por millón } \\
\text { de habitantes }\end{array}$ & $\begin{array}{l}\text { Número de personal de I + D por } \\
\text { millón de habitantes }\end{array}$ & $\begin{array}{l}\text { Instituto de Estadística de UNESCO, } \\
\text { RICYT, oCDE principales Indicadores de } \\
\text { Ciencia y Tecnología, Oficinas Nacio- } \\
\text { nales de Estadística }\end{array}$ \\
\hline ENROL\%_3 & $\begin{array}{l}\text { La matrícula escolar, terciario } \\
\text { (\% bruto) }\end{array}$ & $\begin{array}{l}\text { Enrolamiento: La matrícula escolar, } \\
\text { terciario (\% bruto) }\end{array}$ & Banco Mundial \\
\hline ENROL\%_2 & $\begin{array}{l}\text { La matrícula escolar, secundaria } \\
\text { (\% bruto) }\end{array}$ & $\begin{array}{l}\text { Enrolamiento: La matrícula escolar, } \\
\text { secundaria (\% bruto) }\end{array}$ & Banco Mundial \\
\hline ENROL\%_1 & $\begin{array}{l}\text { La matrícula escolar, primario } \\
\text { (\% bruto) }\end{array}$ & $\begin{array}{l}\text { Enrolamiento: La matrícula escolar, } \\
\text { primario (\% bruto) }\end{array}$ & Banco Mundial \\
\hline PAGOSLICENCIASj,t & $\begin{array}{l}\text { Los pagos per cápita de las re- } \\
\text { galías y licencias }\end{array}$ & $\begin{array}{l}\text { Valor de las regalías y licencias } \\
\text {-pagos per cápita- (PPA) }\end{array}$ & Banco Mundial \\
\hline \multirow[t]{2}{*}{ IMPORTACIONESj,t } & $\begin{array}{l}\text { Las importaciones de bienes de } \\
\text { capital per cápita }\end{array}$ & $\begin{array}{l}\text { Valor de los bienes de capital im- } \\
\text { portar per cápita (PPA) }\end{array}$ & UNCTAD, un Comtrade, Global \\
\hline & & & Trade Atlas \\
\hline APERTURAj,t & Nivel de apertura & $\begin{array}{l}\text { Importaciones más exportaciones } \\
\text { dividido por el producto interno } \\
\text { bruto real por labor (a precios } \\
\text { constantes) }\end{array}$ & Penn World Table (PWT 7.1) \\
\hline
\end{tabular}




\begin{tabular}{|l|l|l|l|}
\hline \multicolumn{4}{|c|}{ VARIABLES INDEPENDENTES (X) } \\
\hline IEDCAPj,t & $\begin{array}{l}\text { La inversión extranjera directa } \\
\text { per cápita }\end{array}$ & $\begin{array}{l}\text { La inversión extranjera directa per } \\
\text { cápita (PPA) }\end{array}$ & Banco Mundial (wDI) \\
\hline PIBCAPj,t & PIB per cápita & $\begin{array}{l}\text { El producto interno bruto per cápi- } \\
\text { ta (PPA) }\end{array}$ & Penn World Table (PWT 6.2) \\
\hline EDUCA\%PIB & $\begin{array}{l}\text { El gasto público en educación } \\
\text { como porcentaje del PIB }\end{array}$ & $\begin{array}{l}\text { El gasto público en educación co- } \\
\text { mo porcentaje del piB comprende el } \\
\text { gasto total público (corriente y de } \\
\text { capital) en educación como por- } \\
\text { centaje del Producto Interno Bruto } \\
\text { (PIB) en un año determinado. }\end{array}$ & \\
\hline
\end{tabular}

Fuente: Elaboración propia con base en Stern, Porter y Furman, (2000); Baumert, Thomas, Heijs y Joost, (2002); Ulku, (2004); Chinaprayoon, (2007); Hu y Mathews, (2008); Gans y Hayes, (2009); CEPAL, (2007); García, Blázquez y López (2012).

Nota: United States Patent and Trademark Office (USPTO, por sus siglas en inglés).

\section{II.2. Alcance del estudio}

De acuerdo con el Banco Mundial (2015) hay 28 países latinoamericanos en vías de desarrollo, lo que representa la población estadística del presente estudio; sin embargo, sólo 17 países producen estadísticas completas ${ }^{5}$ sobre las variables requeridas de la capacidad tecnológica. Por lo tanto, estas 17 economías de ingresos medios (medios-altos y medios-bajos) se utilizarán como muestra dentro del análisis. Además, debido a la limitación de las estadísticas internacionales de I + D, las estadísticas disponibles son compatibles a partir del año 2000. Como resultado, la serie de tiempo cubrirá del 2000 al 2014.

La muestra del estudio contiene a los países de renta media superior e inferior en América Latina, según la clasificación de países del Banco Mundial en el año 2013 (véase cuadro 4). Este documento no incluye a los países de bajos ingresos de América Latina, debido a la falta de estadísticas, y además, de acuerdo con Chinaprayoon (2007), "los países de bajos ingresos son por lo general contribuyentes insignificantes". Por lo tanto, se procede a utilizar una muestra de 17 países (economías de renta media).

${ }^{5}$ Siguiendo a Chinaprayoon (2007), los valores faltantes en algunas de las variables son sustituidos por su valor de tendencia. 
Cuadro 4. Muestra estadistica: lista de os países de la muestra por clasificación de ingresos

\begin{tabular}{|l|l|l|}
\hline Ingreso Medio Alto & Ingreso Medio Bajo \\
\hline Argentina & México & Bolivia \\
\hline Brasil & Panamá & El Salvador \\
\hline Chile & Perú & Guatemala \\
\hline Colombia & República Dominicana & Honduras \\
\hline Costa Rica & Uruguay & Paraguay \\
\hline Ecuador & Venezuela & \\
\hline
\end{tabular}

Fuente: Elaboración propia con base en Banco Mundial (2015).

\section{ANÁLISIS DE LOS RESULTADOS DESCRIPTIVOS Y ECONOMÉTRICOS}

\section{III.1. Estadística descriptiva}

El análisis descriptivo es necesario para examinar las propiedades estadísticas de cada variable y, de acuerdo con Chinaprayoon (2007), poder proponer las variables que serán usadas para evaluar el impacto de la capacidad tecnológica en los países seleccionados.

En la estadística descriptiva de las variables previamente normalizadas por el tamaño de la población, se reporta la media, la desviación estándar y el coeficiente de variación, esto agrupado en el cuadro 5, algunos de los principales hallazgos se describen a continuación:

Los resultados logrados: En América Latina, en promedio cada país de la muestra produce 21 patentes USPTO por año, en otras palabras en Latinoamérica se produce media patente (.52) por cada millón de habitantes al año. En dicho caso, el coeficiente de variación es elevado, por lo que se asume una alta dispersión de los datos con respecto a la media, adjudicando dicha dispersión, a las notorias diferencias de la dinámica de patentamiento entre los países de ingreso medio alto y medio bajo. Los resultados descriptivos, también indican que en promedio se realizan dos solicitudes de patente y 26 publicaciones científicas por cada millón de habitantes al año. En promedio 8.6 por ciento de las exportaciones anuales de manufactura son exportaciones de alta tecnología. Mientras que los ingresos (cobros promedio) por el uso de propiedad intelectual ascienden a 1.5 dólares por habitante al año.

La base disponible: Se estima que en promedio el stock inicial de patentes en Latinoamérica es de 450 patentes USPTO. Existen también en promedio 328 investigadores $\mathrm{I}+\mathrm{D}$ por cada millón de habitantes latinoamericanos. Con lo que respecta a la tasa de enrolamientos, el terciario es el más bajo con un promedio de 38 por 
ciento, le sigue el secundario con una cobertura media de 77 por ciento y el enrolamiento primario muestra una matrícula media mayor a 100 por ciento.

Los esfuerzos realizados: En lo que respecta, por ejemplo al Gasto en Investigación y Desarrollo, los países de toda la muestra financian dichas actividades con .30 por ciento del PIB anualmente. En este caso existen notorias diferencias entre los países latinoamericanos de ingreso medio alto y medio bajo, los primeros manejan un porcentaje promedio de inversión de .41 por ciento, mientras que los segundos únicamente .08 por ciento. El gasto en educación (como porcentaje del PIB) promedio es de 4 por ciento y los egresos (pagos promedio) por el uso de propiedad intelectual ascienden a 11.7 dólares por habitante al año, lo que inicialmente corrobora la postura de que las firmas en países en desarrollo generan sus procesos de aprendizaje que subyacen la construcción y acumulación gradual de capacidades tecnológicas a partir de la tecnología que adquieren de firmas en otros países.

Cuadro 5. Estadística descriptiva: América Latina (toda la muestra)

\begin{tabular}{|c|c|c|c|c|}
\hline Variables & $\mathrm{N}$ & Media & Desviación Estándar & Coeficiente de Variación \\
\hline \multicolumn{5}{|l|}{ Resultados logrados (Y) } \\
\hline Patentes otorgadas & 255 & 21.21 & 43.87 & 206.87 \\
\hline Patentes otorgadas por millón habitantes & 255 & 0.5223 & 0.6463 & 123.73 \\
\hline Solicitudes de patentes & 235 & 65.39 & 129.34 & 197.79 \\
\hline Solicitudes de patentes por millón habitantes & 235 & 1.669 & 1.791 & 107.33 \\
\hline Publicaciones científicas & 255 & 1298 & 2850 & 219.48 \\
\hline Publicaciones científicas por millón de habitantes & 255 & 25.66 & 32.26 & 125.70 \\
\hline Exportaciones de alta tecnología per cápita & 254 & 63.94 & 150.89 & 235.98 \\
\hline Exportaciones de manufactura de alta tecnología (\%) & 254 & 8.640 & 9.837 & 113.85 \\
\hline Cobros de licencias per cápita & 114 & 1.538 & 2.101 & 136.66 \\
\hline \multicolumn{5}{|l|}{ La base disponible $(X)$} \\
\hline Stock de patentes & 255 & 450.4 & 762.7 & 169.34 \\
\hline Electricidad per cápita & 255 & 1551.8 & 879.3 & 56.67 \\
\hline Teléfono por 100 habitantes & 255 & 15.499 & 7.150 & 46.14 \\
\hline Celular por 100 habitantes & 255 & 69.31 & 46.35 & 66.87 \\
\hline Internet por 100 habitantes & 255 & 22.50 & 16.77 & 74.55 \\
\hline PIB per cápita & 255 & 9939 & 4371 & 43.97 \\
\hline Investigadores I+D por millón habitantes & 179 & 328.4 & 339.3 & 103.33 \\
\hline Apertura (exportaciones + importaciones/PIB) & 255 & 69.70 & 30.04 & 43.10 \\
\hline Importaciones bienes per cápita & 170 & 1703.6 & 1200.4 & 70.46 \\
\hline Enrolamiento primario (\% bruto) & 240 & 108.95 & 6.50 & 5.97 \\
\hline Enrolamiento secundario (\% bruto) & 234 & 77.775 & 13.574 & 17.45 \\
\hline Enrolamiento terciario (\% bruto) & 227 & 38.10 & 16.40 & 43.05 \\
\hline
\end{tabular}


Cuadro 5. Continuación

\begin{tabular}{|l|r|r|r|r|}
\hline Los esfuerzos realizados (X) & & & & \\
\hline Gasto público en educación (\% PIB) & 226 & 4.0379 & 1.3260 & 32.84 \\
\hline Gasto en investigación y desarrollo (\%PIB) & 192 & 0.3073 & 0.2822 & 91.85 \\
\hline Inversión Extranjera Directa per cápita & 255 & 217.5 & 269.6 & 123.96 \\
\hline Pagos de licencias per cápita & 170 & 11.72 & 13.63 & 116.30 \\
\hline
\end{tabular}

Fuente: Elaboración propia (spss 21).

\section{III.2. Modelo Econométrico: Especificaciones}

En primer lugar, es importante recalcar que los parámetros asociados con los modelos de regresión resultantes en esta sección se evalúan utilizando un conjunto de panel de datos para 17 países latinoamericanos a lo largo de 14 años. Por tanto, tal como apuntan Stern y Porter (2002), estas estimaciones pueden depender de la variación de la sección transversal, es decir, la variación de series de tiempo o de espacio (países), o ambos ${ }^{6}$. Se percibe entonces, que el enfoque más simple para analizar datos tipo panel es omitir las dimensiones del espacio y el tiempo de los datos agrupados y sólo calcular la regresión MCO usual. Sin embargo, otra manera mucho más precisa de modelar el carácter individual de cada entidad es a través del modelo de efectos fijos y es precisamente este último el que se utilizará 7 para la presente investigación, habiendo de comprobar estadísticamente su pertinencia.

La ventaja fundamental de un panel de datos con efectos fijos es que nos permite estudiar un gran abanico de temas económicos que no podrían abordarse usando solamente modelos de series de tiempo o de corte transversal. En particular, los datos de panel permiten tener en cuenta la existencia de efectos individuales inobservables, que pueden estar correlacionados con otras variables incluidas en la especificación de una relación econométrica. Proveen al investigador un gran número de datos, proporcionando menos problemas de multicolinealidad (presente en numerosas ocasiones al utilizar datos de series temporales), más grados de

${ }^{6}$ Para Stern, Porter, y Furman (2000), en la mayoría (pero no todos) de sus análisis, incluyeron, ya sea variables ficticias (dummies) de año o una tendencia temporal, con el fin de explicar las diferencias en evolución a través de años en el nivel general de los resultados innovadores. Mucho de su análisis también incluye variables ficticias (dummies) de los países para controlar las diferencias en la sofisticación tecnológica (por ejemplo, como se refleja en el PIB per cápita).

${ }^{7}$ En los casos en los que los efectos fijos resulten estadísticamente pertinentes/viables después de las pruebas de especificación bajo el criterio del investigador. 
libertad y, por tanto, mayor eficiencia de las estimaciones. Su utilización permite recoger con mayor precisión la variabilidad en los datos, tanto la existente entre individuos como la que existe a lo largo del tiempo. La gran ventaja de los datos de panel es que tienen en cuenta las diferencias permanentes entre individuos, aunque éstas no se observen a simple vista (Franco, Ramos y Hernández 2016).

Aparicio y Márquez (2005) apuntan que el modelo de panel de datos con efectos fijos no supone que las diferencias entre entidades sean aleatorias, sino constantes o fijas y por ello se debe estimar cada intercepto $v_{i}$ (country_dummy) haciendo uso de la técnica de las variables dicotómicas de intersección diferencial. Donde $v_{i}$ es un vector de variables dicotómicas (o variables dummy) para cada entidad.

De acuerdo con numerosos autores (Aparicio y Márquez, 2005; Mayorga y Muñoz, 2008; Labra y Torrecillas, 2013; Torres, 2007; Montero, 2011), para definir modelos econométricos con datos tipo panel, es necesario realizar una serie de pruebas de especificación. Primero es conveniente cuestionarse: ¿cuándo se debe aplicar un MCO agrupado y cuándo un modelo de datos anidados? Y en este último caso, identificar si los efectos fijos son más convenientes que los aleatorios. Para ello, inicialmente se debe verificar que la varianza de $v_{i}$ (del modelo anidado) sea significativamente distinta de cero, para dichos efectos, se corre el test de Breusch-Pagan, también denominado del Multiplicador de Lagrange. La prueba consiste en realizar la regresión auxiliar indepit $=d_{e p} i t+u_{i}+e_{i t}$. La hipótesis nula es Var $\left(u_{i}\right)=0$ con una $\mathrm{chi}^{2}$ de contraste $^{8}$. Si el valor del test es bajo ( $\mathrm{p}$-valor mayor de 0.95 ) la hipótesis nula se confirma y es mejor MCO. Si el valor del test es alto (p-valor menor de 0.05), la hipótesis nula se rechaza y es mejor elegir un modelo anidado. En otras palabras si se rechaza la hipótesis nula, implicaría que efectivamente existe un componente inobservable de la varianza asociada a cada individuo.

Como segundo paso, es necesario comparar las estimaciones del modelo de efectos fijos y el de efectos aleatorios. Para ello, se realiza el test de Hausman, en el cual, si se encuentran diferencias sistemáticas (se rechaza la hipótesis nula de igualdad, es decir se obtiene un valor de la prueba alto y un p-valor bajo, menor de $0.05)$, entonces se podrá asumir que continúa existiendo correlación entre el error y los regresores $(\operatorname{Cov}($ Xit,uit $) \neq 0$ ) y es preferible elegir el modelo de efectos fijos al modelo de efectos aleatorios. En un tercer paso, Aparicio y Márquez (2005), señalan que es conveniente realizar la prueba Test Parm, para verificar si, también es posible agregar variables dicotómicas temporales al modelo (con notación $\eta_{\mathrm{t}}$ ), es decir, una year_dummy para cada año en la muestra, que capturen eventos co-

${ }^{8} \mathrm{El} \mathrm{prob}>\mathrm{chi}^{2}>0.05$. 
munes a todos los estados durante un período u otro. En donde la hipótesis nula es: $\eta_{1}=\eta_{2}=\ldots=\eta_{t}=0$, en ese sentido, el $p$-value de la prueba $F$ indica que sí rechazamos la hipótesis nula, se podría afirmar que las variables dicotómicas temporales son conjuntamente significativas y pertenecen al modelo, de modo contrario se acepta la $\mathrm{H}_{0}$, lo que implicaría que los efectos fijos temporales serían estadísticamente insignificantes. Finalmente, se requiere correr las pruebas de Wooldridge y Wald, en las cuales, si rechazamos la hipótesis nula, evidenciamos problemas de autocorrelación y heterocedasticidad, los cuales deberán solucionarse conjuntamente con estimadores de Mínimos Cuadrados Generalizados Factibles (Feasible Generalizad Least Squares o FGLS), o bien con Errores Estándar Corregidos para Panel (Panel Corrected Standard Errors o PCSE).

Es importante mencionar que además de las pruebas de especificación, el criterio del investigador ${ }^{9}$ y las características de la muestra ${ }^{10}$, son determinantes para la elección de un modelo de regresión agrupado (MCO) en comparación con uno anidado (de efectos fijos o aleatorios). De acuerdo con Franco, Ramos, y Hernández (2016), no siempre se muestra de forma evidente qué modelo debe emplearse en un caso u otro, por lo que es esencial considerar que si se desea hacer inferencias con respecto a la población, es decir que se trabaja con una muestra aleatoria, se recomienda el uso de un modelo con efectos aleatorios. Por el contrario si se trabaja con una muestra seleccionada a conveniencia se recomienda un modelo con efectos fijos. Además, éste último es ampliamente útil en el caso de que el número de unidades de análisis o de instantes de tiempo no sea grande. Por el contrario, en el caso de que se disponga de un número grande de observaciones a través del tiempo se recomienda un modelo de efectos aleatorios.

Consecuentemente, dado que en el presente trabajo la muestra estadística se seleccionó a conveniencia (países latinoamericanos) y tanto la serie temporal (14 años) como la dimensión transversal (17 países) no es grande, se considera prudente optar por un modelo de regresión con efectos fijos (predilección), sin embargo, en los casos en que no se rechace la hipótesis nula de la prueba Hausman y que por consecuencia estadísticamente sea preferible el modelo con efectos aleatorios, se antepondrá el criterio del investigador para entonces optar por un

${ }^{9}$ Los criterios en investigaciones previas aconsejan la estimación de modelos con efectos fijos (Stern, Porter, y Furman, 2000; Baumert, Thomas, Heijs y Joost, 2002; Ulku, 2004; Chinaprayoon, 2007; Hu y Mathews, 2008; Gans y Hayes, 2009).

${ }^{10}$ El modelo de efectos fijos se ve como un caso en que el investigador hace inferencia condicionada a los efectos que ve en la muestra. El de efectos aleatorios se ve como uno en el cual el investigador hace inferencia condicional o marginal respecto a una población. Se deja al investigador que decida si hace inferencia con respecto a las características de una población o sólo respecto a los efectos que están en la muestra (Mayorga y Muñoz 2000). 
Figura 1. Pruebas estadísticas y modelo óptimo final

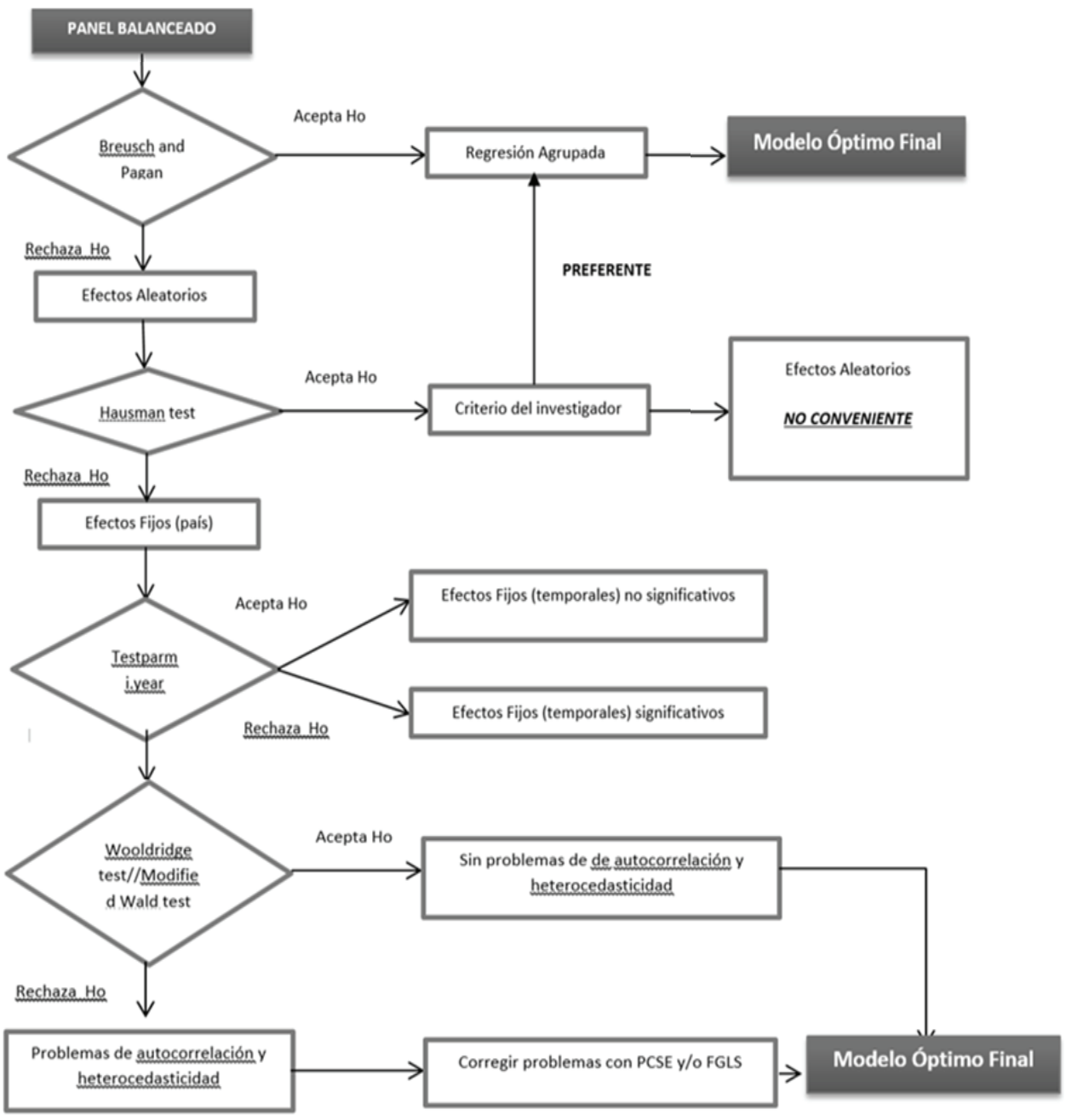

Nota: La Ho se rechaza si el p-value de la prueba es menor a 0.05.

Fuente: Elaboración propia con base en Aparicio y Márquez (2005); Mayorga y Muñoz (2008); Labra y Torrecillas (2013); Torres (2007), y Montero (2011).

modelo agrupado MCO. Lo anteriormente descrito, se ilustra en el siguiente flujograma (figura 1), el cual contiene el procedimiento de las pruebas de especificación de los modelos de regresión resultantes en nuestra investigación. 


\section{III.3. Modelo econométrico: resultados}

De entrada, se presenta la matriz de correlación entre variables independientes (cuadro 6) con la finalidad de evitar problemas de multicolinealidad en el modelo, ya que de acuerdo con Labra y Torrecillas (2013), la selección de variables a incluir en el modelo dependerá no sólo de la información que la literatura especializada pueda entregar, sino que además, se recomienda hacer un análisis de correlación, con el fin de excluir las variables que tengan un mismo comportamiento ${ }^{11}$.

Bajo dicho tratamiento, se evidencia un comportamiento similar entre:

- El Gasto en $\mathrm{I}+\mathrm{D}\left(\mathrm{X}_{6}\right)$ y el número de investigadores $\left(\mathrm{X}_{7}\right)$.

- Las importaciones $\left(\mathrm{X}_{9}\right)$ y la IED $\left(\mathrm{X}_{11}\right)$. Ambas son indicadores de la adquisición de conocimiento externo.

- El consumo de electricidad $\left(\mathrm{X}_{2}\right)$ tiene una alta correlación con muchas variables incluyendo a las líneas telefónicas $\left(\mathrm{X}_{3}\right)$. Ambas son indicadores de la difusión de las viejas tecnologías. Por lo tanto eliminamos la variable $\left(\mathrm{X}_{2}\right)$ y seleccionamos $\left(\mathrm{X}_{3}\right)$.

- El pago de licencias $\left(\mathrm{X}_{8}\right)$ y el stock de patentes $\left(\mathrm{X}_{1}\right)$.

- Pagos de licencias $\left(\mathrm{X}_{8}\right)$ e IED $\left(\mathrm{X}_{11}\right)$.

- Stock de patentes $\left(\mathrm{X}_{1}\right)$ y Gasto en $\mathrm{I}+\mathrm{D}\left(\mathrm{X}_{6}\right)$.

- Internet $\left(\mathrm{X}_{5}\right)$ y IED $\left(\mathrm{X}_{11}\right)$

Derivado de lo anterior, se detectaron las variables que estando juntas en un mismo modelo, ${ }^{12}$ lo sobrexplican, por tal motivo se decidió diseñar dos modelos de regresión (A y B) para cada variable dependiente, de tal modo, que las variables independientes de cada modelo, incluyan indicadores de la base disponible y de los esfuerzos realizados, pero evitando problemas de multicolinealidad. En otras palabras, tratando de aprovechar la mayoría de nuestros indicadores, se buscó que las variables correlacionadas no se incluyan en el mismo modelo, por lo que fue necesario construir dos modelos que expliquen la capacidad tecnológica latinoamericana pero con regresores diferentes. Las variables de dichos modelos se pueden apreciar en el cuadro 7 .

${ }^{11}$ Bajo el criterio generalmente utilizado en esta clase de investigaciones de $\mathrm{R} \geq 0.70$.

${ }^{12}$ Modelo de la forma (5): Los resultados logrados $=f$ (La base disponible, los esfuerzos realizados). 
Cuadro 6. Matriz de correlación entre variables

\begin{tabular}{|r|rrrrrrrrrrrr} 
& $x 1$ & $x 2$ & $x 3$ & $x 4$ & $x 5$ & $x 6$ & $x 7$ & $x 8$ & $x 9$ & $x 10$ & $x 11$ & $x 13$ \\
\hline$x 1$ & 1.0000 & & & & & & & & & & \\
$x 2$ & 0.5131 & 1.0000 & & & & & & & & & \\
$x 3$ & 0.5793 & 0.7274 & 1.0000 & & & & & & & & \\
$x 4$ & 0.0710 & 0.2433 & 0.0748 & 1.0000 & & & & & & & \\
$x 5$ & 0.3612 & 0.7091 & 0.5109 & 0.5820 & 1.0000 & & & & & & & \\
$x 6$ & 0.7457 & 0.6064 & 0.5735 & 0.0394 & 0.4383 & 1.0000 & & & & & & \\
$x 7$ & 0.6562 & 0.7055 & 0.6811 & 0.0776 & 0.5520 & 0.7359 & 1.0000 & & & & & \\
$x 8$ & 0.6498 & 0.6282 & 0.6887 & 0.3514 & 0.5929 & 0.4920 & 0.5084 & 1.0000 & & & & \\
$x 9$ & 0.0100 & 0.5958 & 0.3203 & 0.4077 & 0.5565 & -0.0485 & 0.1213 & 0.4548 & 1.0000 & & & \\
$x 10$ & -0.6586 & -0.0159 & -0.3335 & 0.0131 & -0.0347 & -0.5510 & -0.3887 & -0.1648 & 0.6186 & 1.0000 & & \\
$x 11$ & 0.3580 & 0.6760 & 0.6689 & 0.3465 & 0.6036 & 0.3408 & 0.4563 & 0.6882 & 0.6556 & 0.0901 & 1.0000 & \\
$x 13$ & 0.3094 & -0.0806 & -0.0426 & -0.2023 & 0.1936 & 0.3990 & 0.4012 & 0.0581 & -0.3142 & -0.2963 & -0.1180 & 1.0000
\end{tabular}

Fuente: Elaboración propia (Stata 12).

Cuadro 7. La base de los modelos de regresión $A$ y $B$

\begin{tabular}{|c|c|c|c|c|c|}
\hline \multirow[t]{2}{*}{ Dimensión } & & Modelo A & \multirow[t]{2}{*}{ Dimensión } & \multicolumn{2}{|r|}{ Modelo B } \\
\hline & \multicolumn{2}{|c|}{ Variables Independientes } & & \multicolumn{2}{|c|}{ Variables Independientes } \\
\hline \multirow{5}{*}{ BD } & \multicolumn{2}{|c|}{ Capacidades tecnologicas de infraestructura } & \multirow{8}{*}{$\mathrm{BD}$} & \multicolumn{2}{|c|}{ Capacidades tecnologicas de infraestructura } \\
\hline & $x 3$ & Telefono (Ln) & & $\mathrm{X} 1$ & Stock de patentes (Ln) \\
\hline & $\times 5$ & Internet (Ln) & & $\mathrm{X} 2$ & Telefono (Ln) \\
\hline & \multicolumn{2}{|c|}{ "Inserción comercial internacional" } & & X3 & Celular (Ln) \\
\hline & $\mathrm{X} 10$ & Apertura (Ln) & & \multicolumn{2}{|c|}{$\begin{array}{l}\text { Capacidades de absorción } \\
\text { (acervo de recursos humanos) }\end{array}$} \\
\hline \multirow{8}{*}{ ER } & \multicolumn{2}{|c|}{ Inputs de absorción } & & $X 7$ & Investigadores I+D (Ln) \\
\hline & $\mathrm{X} 13$ & Gasto en educación\%PIB & & \multicolumn{2}{|c|}{ Inserción comercial internacional } \\
\hline & \multicolumn{2}{|c|}{ Inputs de innovación } & & $\mathrm{X} 10$ & Apertura (Ln) \\
\hline & $x 6$ & GIDE \% PIB & \multirow{5}{*}{ ER } & \multicolumn{2}{|c|}{ Inputs de absorción } \\
\hline & \multirow{2}{*}{\multicolumn{2}{|c|}{$\begin{array}{l}\text { Inputs Tecnologicos } \\
\text { (adquisición de conocimiento externo) }\end{array}$}} & & $\mathrm{X} 13$ & Gasto en educación \%PIB \\
\hline & & & & \multirow{2}{*}{\multicolumn{2}{|c|}{$\begin{array}{l}\text { Inputs Tecnologicos } \\
\text { (adquisición de conocimiento externo) }\end{array}$}} \\
\hline & $\mathrm{x} 8$ & Pago de licencias (Ln) & & & \\
\hline & X9 & Importaciones de bienes ( $(\mathrm{n})$ & & $\mathrm{X} 11$ & $\operatorname{IED}(\operatorname{Ln})$ \\
\hline
\end{tabular}

Nota: (Ln) denota Logaritmo Natural. BD= Base Disponible, ER= Esfuerzos realizados. Para el modelo A y $B$, las variables dependientes son: $Y 1=$ PATENTES OTORGADAS (Ln), Y2= SOLICITUDES DE PATENTE (Ln), Y3= PUBLICACIONES CIENT (Ln), Y4= PIB PER CÁPITA (Ln).

Fuente: Elaboración propia. 
Se observa entonces que los regresores del modelo B surgen dado que las variables del modelo A pueden ser sustituidas unas por otras. Futuros estudios deberán hacer la elección de un solo modelo de regresión si el objetivo fuese la estimación de indicadores sintéticos.

\section{III.3.1. Modelo de panel de datos con efectos fijos}

El modelo preferente de panel de datos con efectos fijos queda denotado de la siguiente manera:

$$
\begin{aligned}
& Y_{i t}=\beta_{0}+\sum_{i=1}^{N-1} \mu_{i} v_{i}+\sum_{t=1}^{T-1} \partial_{t} \eta_{t}+\sum_{k=1}^{k} \beta_{k} \mathrm{X}_{k i t}+e_{i t} \\
& \mathrm{i}=1, \ldots, \mathrm{N} \quad \mathrm{t}=1, \ldots, \mathrm{T} \quad \mathrm{k}=1, \ldots, \mathrm{K}
\end{aligned}
$$

\begin{tabular}{|c|c|c|}
\hline \multicolumn{2}{|r|}{ MODELOA } & MODELO B \\
\hline$Y_{i t}$ & \multicolumn{2}{|c|}{$Y_{1}:$ LPATENTES $_{i, t} Y_{2}:$ LPATENTES_APLI $\mathrm{i}_{\mathrm{i}, \mathrm{t}} \mathrm{Y}_{3}:$ LPUBLICACIONES $_{i, t_{t}} \quad Y_{4}:$ LPIBCAP $_{i, t}$} \\
\hline i & \multicolumn{2}{|l|}{ Países o unidades de estudio: 17 países latinoamericanos } \\
\hline $\mathrm{t}$ & \multicolumn{2}{|l|}{ Observaciones en el tiempo: 14 años (2000-2014) } \\
\hline$\beta_{0}$ & \multicolumn{2}{|l|}{ Vector de interceptos de $n$ parámetros } \\
\hline$\beta$ & \multicolumn{2}{|l|}{ Vector de k parámetros. } \\
\hline $\mathrm{k}$ & Variables explicativas: $L X_{3}, L X_{5}, L X_{10}, L X_{13}, X_{6}, L X_{8}, L X_{9}$ & Variables explicativas: $\mathrm{LX}_{1}, \mathrm{LX} \mathrm{X}_{3}, \mathrm{LX}_{4}, \mathrm{LX} \mathrm{X}_{7} \mathrm{X}_{10}, \mathrm{LX}_{13}, \mathrm{LX} \mathrm{X}_{11}$ \\
\hline $\mathrm{X}_{\text {kit }}$ & \multicolumn{2}{|c|}{ i-ésima observación al momento t para la variable explicativa k } \\
\hline$e_{i t}$ & \multicolumn{2}{|c|}{ Término de error que representa los efectos de todas las variables omitidas en el modelo } \\
\hline$\mu_{i} v_{i}$ & \multicolumn{2}{|l|}{ Variable dicotómica de efectos fijos (COUNTRY_DUMMY) } \\
\hline$\partial_{t} \eta_{t}$ & \multicolumn{2}{|c|}{ Variable dicotómica de efectos fijos temporales (YEAR_DUMMY). } \\
\hline
\end{tabular}

Donde:

Nota: (L) denota Logaritmo Natural.

Fuente: Elaboración propia. 
Para la base del modelo A y B, se establece una ecuación de regresión de datos de panel con efectos fijos por lo que en este caso $\mu_{\mathrm{i}}$ capta las diferencias estructurales entre unidades muestrales (países) por medio de los N-1 términos independientes adicionales. Las diferencias en instantes de tiempo (años) son captadas a través de $\delta_{\mathrm{t}}$. Para este modelo $\beta_{0}$ es el término independiente para la unidad individual cuyos términos $\mu_{\mathrm{i}} \mathrm{y} \delta_{\mathrm{t}}$ se han excluido. Como se observa en los cuadros 8 y 9 , la ecuación de regresión (6), se aplica en todos $\operatorname{los} \operatorname{casos}^{13}$ a excepción de cuando la variable explicada es $\mathrm{Y}_{1}$ (para el modelo A) y $\mathrm{Y}_{3}$ (para el modelo B), en cuyas excepciones, se opta por un modelo de regresión con MCO. ${ }^{14}$

Cuadro 8. Resultados Modelo A

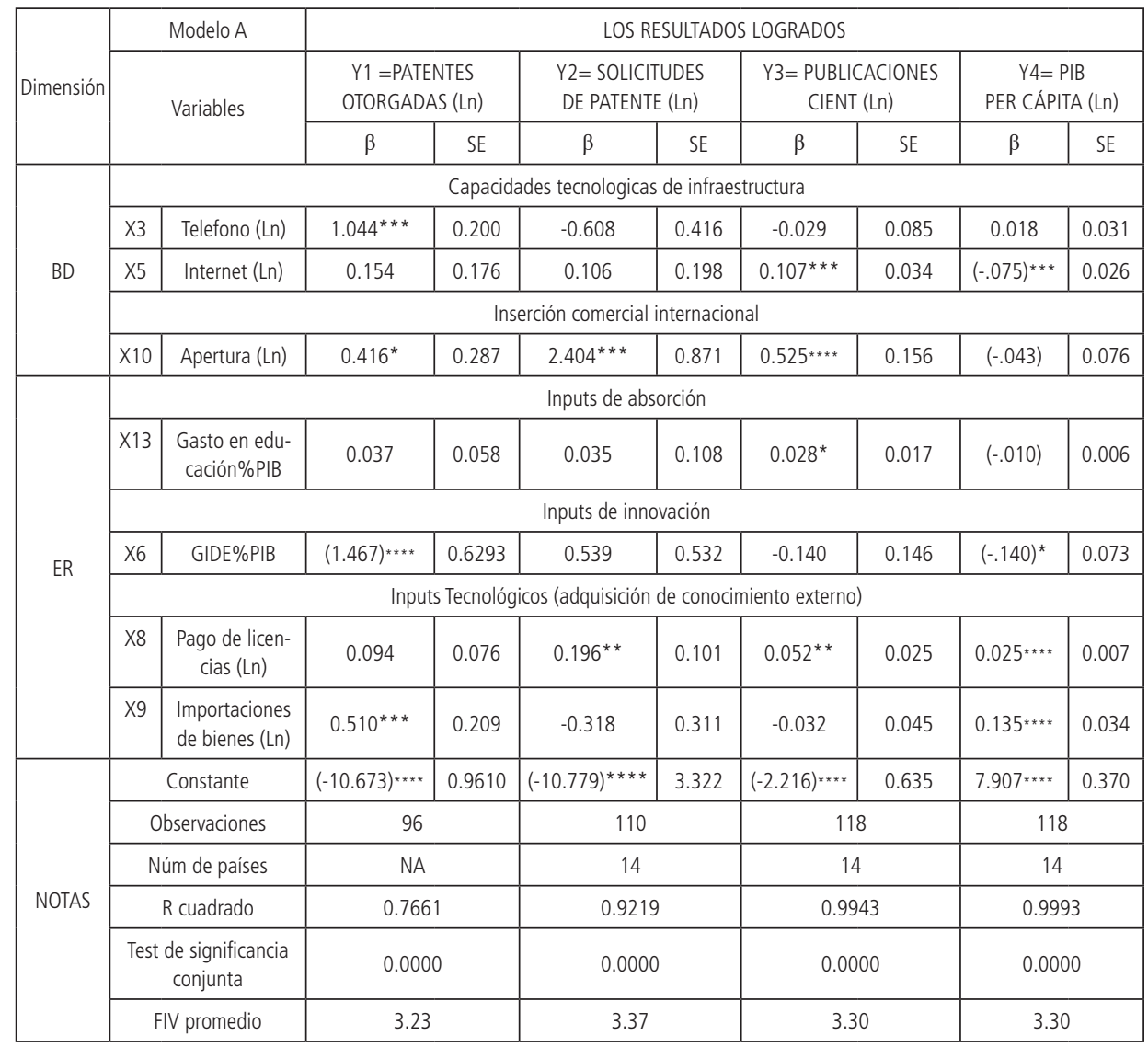

${ }^{13}$ En algunos casos se requiere considerar variables dicotómicas para los países y/o variables dicotómicas temporales (dummy_country y/o dummy_year).

${ }^{14}$ Bajo la ecuación: $Y_{i t}=\bar{\alpha}+\beta_{1} X_{1 t}+e_{t}$ 
Cuadro 8. Continuación

\begin{tabular}{|c|c|c|c|c|c|}
\hline $\begin{array}{c}\text { Breusch and Pagan La- } \\
\text { gragian MTRE }\end{array}$ & 0.0000 & 0.0001 & 0.0000 & 0.0000 \\
\cline { 2 - 6 } & Hausman test & 0.8976 & 0.0031 & 0.0000 & 0.0003 \\
\cline { 2 - 6 } & Efectos fijos de paises & No significativos & Significativos & Significativos & Significativos \\
\hline Testparm i.year & 0.4116 & 0.2191 & 0.2005 & 0.0000 \\
\hline Efectos fijos de tiempo & No significativos & No significativos & No significativos & Significativos \\
\hline Wooldridge test & NA & 0.2745 & 0.7657 & 0.0000 \\
\hline Modified Wald test het & NA & 0.0000 & 0.0000 & 0.0000 \\
\hline & Modelo Óptimo Final & MCO & PCSE_corregido & FGLS_corregido & PCSE_corregido \\
\hline
\end{tabular}

NOTAS ADICIONALES:

Abreviaturas: $\mathrm{BD}=$ Base Disponible; $\mathrm{ER}=$ Esfuerzos Realizados. $\mathrm{MCO}=$ Mínimos Cuadrados Ordinarios. PCSE $=$ Panel Corrected Standard Errors. FGLS= Feasible Generalizad Least Squares . (Ln) = Logaritmo Natural. NA = No Aplica. SE= Error Estándar. FIV promedio = Factor de Inflación de la Varianza (cuando el valor de FIV es mayor a 10, se puede sospechar de problemas de multicolinealidad).

Significancia del: ${ }^{*} p \leq 0.10 \quad * * p \leq 0.05 \quad * * * p \leq 0.01 \quad * * * * p \leq 0.001$

Fuente: Elaboración propia con base en resultados procesados en (Stata 12).

Figura 2. Elasticidades de los factores significativos del Modelo A

0.03

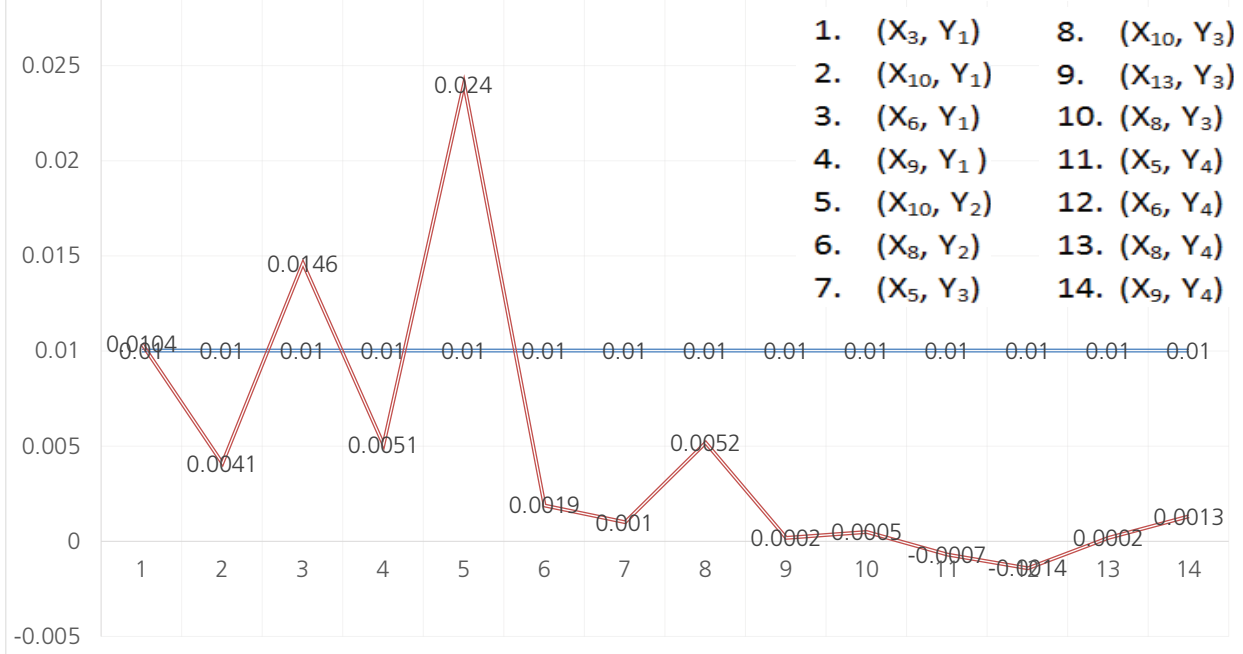

Fuente: Elaboración propia con base en resultados.

Consecuentemente con lo anterior, se presentan las gráficas tanto del modelo A como para el modelo B, que muestran visualmente los factores de la capacidad tecnológica que resultaron estadísticamente significativos y su respectiva elastici- 
dad. En otras palabras, estas figuras muestran las "piezas" que un tomador de decisiones puede mover a sabiendas de los impactos que tendrán sobre la capacidad tecnológica. En donde los efectos elásticos son aquellos que están por encima de la línea horizontal, es decir, que manifiestan variaciones proporcionalmente mayores al insumo; contrariamente, los efectos inelásticos se encuentran por debajo de esta línea y manifiestan variaciones proporcionalmente menores al insumo (véase figuras 2 y 3 ).

\section{III.3.2. Interpretaciones Modelo A}

De acuerdo con las estimaciones del modelo A, se hace la interpretación ${ }^{15}$ de los coeficientes que resultaron estadísticamente significativos (véase cuadro 8). La lectura de los hallazgos se realiza mediante seis enunciados que señalan la elasticidad de las variables explicadas (de capacidad tecnológica) ante cambios del 1 por ciento de los regresores (de la base disponible y de los esfuerzos realizados).

- Si el gasto en I+D se incrementa en 1 por ciento, se espera que las patentes otorgadas (USPTO) aumenten 1.46 por ciento y el PIB per cápita disminuya en .14 por ciento.

Lo anterior implica que en Latinoamérica, los esfuerzos realizados en materia de inversión en $\mathrm{I}+\mathrm{D}$, funcionan como un claro determinante de la capacidad en materia de obtención de patentes, inclusive con un porcentaje de elasticidad mayor al de los demás países desarrollados, ya que de acuerdo con las estimaciones de Chinaprayoon (2007), el 1 por ciento de incremento en el gasto en $\mathrm{I}+\mathrm{D}$, lleva a un aumento del .59 por ciento en las patentes otorgadas en países en vías de desarrollo de todo el mundo. Mientras que de, acuerdo con estudios de la Organización para la Cooperación y el Desarrollo Económicos (OCDE), 1 por ciento de aumento en los gastos asociados agregados de I $+\mathrm{D}$ se relacionan con un incremento del 0.07 al 0.1 por ciento en las patentes internacionales (Gans y Hayes, 2005).

El hallazgo encontrado, expresa una buena noticia para la región analizada, dado que entonces, las inversiones en este rubro pronostican materializarse en patentes internacionales otorgadas a una tasa superior a la tasa de gasto. Lo cual concuerda con Chinaprayoon (2007), en el sentido de que un gasto relativamente bajo en I + D dentro de estos países, puede conducir a que cada inversión adicional en las actividades de I $+\mathrm{D}$ impulse el aumento de las patentes internacionales a una tasa más alta que en los países de la OCDE.

${ }^{15}$ La interpretación de los coeficientes se sujeta a la condición Cetiris paribus. Cabe mencionar que en ningún caso se comprueba causalidad. 
- Si las líneas telefónicas se incrementan en 1 por ciento, se espera que las patentes otorgadas aumenten en 1.04 por ciento.

Las líneas telefónicas son un indicador de infraestructura que aporta un conocimiento general del entorno en el cual se desarrollan las actividades productivas, en este caso latinoamericanas; indirectamente esta variable nos ofrece indicios del grado de sofisticación de la producción, "ya que puede suponerse que a mayor valor de los indicadores en cuestión corresponde una mayor sofisticación, lo que debería traducirse en mayor valor agregado en la producción” (CEPAL, 2007). Sin embargo, al ser las líneas telefónicas un factor estadísticamente significativo de la capacidad tecnológica (patentes internacionales), implica que la difusión de las viejas tecnologías, es un elemento predominante en Latinoamérica, por encima de la difusión de las nuevas tecnologías (internet y usuarios de celular).

En otras palabras, la capacidad tecnológica de esta región, principalmente se encuentra forjada gracias a la difusión de las viejas tecnologías.

- Si los usuarios de internet se incrementan en 1 por ciento, se espera que las publicaciones científicas aumenten .10 por ciento y el PIB per cápita decremente en .075 por ciento.

La tasa de penetración de internet no sólo representa la difusión de las nuevas tecnologías dentro de las fronteras nacionales, sino también representa las extensiones de la tecnología a bordo (Chinaprayoon, 2007). En este caso, este indicador tiene un impacto significativo (aunque relativamente bajo) en la generación de publicaciones científicas.

- Si el grado de apertura comercial se incrementa en 1 por ciento, se espera que las patentes otorgadas aumenten 0.41 por ciento, que las solicitudes de patente incrementen 2.4 por ciento y que las publicaciones científicas aumenten también en un 0.52 por ciento.

Lo anterior implica que en Latinoamérica, el grado de apertura (inserción comercial internacional) tiene impactos múltiples y significativos, dado que se relaciona con la obtención de patentes internacionales, solicitudes de patente y publicaciones científicas. Lo que implica entre otras cosas, que existe una fuerte relación entre las fuerzas del mercado internacional y el sector de la ciencia y la tecnología en los países latinoamericanos. 
- Si el gasto en educación se incrementa en 1 por ciento, se espera que las publicaciones científicas aumenten 0.028 por ciento.

El gasto en educación como los esfuerzos realizados de la capacidad de absorción, genera un ligero pero significativo incremento en las publicaciones científicas latinoamericanas.

- Si el pago de licencias se incrementa en 1 por ciento, se espera que las solicitudes de patente aumenten 0.196 por ciento, las publicaciones científicas lo hagan en .052 por ciento y el PIB per cápita se vea incrementado en .025 por ciento.

- Si las importaciones aumentan en 1 por ciento, se espera que las patentes otorgadas se incrementen en 0.51 por ciento y el PIB per cápita aumente en 0.13 por ciento.

El pago de licencias por el uso de la propiedad intelectual tiene impactos significativos en las solicitudes de patente. Además, el pago de licencias y las importaciones tienen ligeros impactos en el PIB, lo cual de alguna manera, es congruente con la literatura que señala que los países en desarrollo (PED) son borrowers o learners, es decir toman prestada y aprenden la tecnología desarrollada por los países desarrollados (Torres, 2006), lo anterior se comprueba sabiendo que al aumentar 1 por ciento de las importaciones es posible que las patentes internacionales se incrementen en medio punto porcentual.

En resumen, los determinantes de la capacidad tecnológica ${ }^{16}$ en Latinoamérica (bajo la base del modelo a), son principalmente el gasto en $\mathrm{I}+\mathrm{D}$, dado que muestra tener un impacto de 1.46 por ciento en la generación de patentes internacionales, por cada 1 por ciento invertido. Otro de los factores determinantes es el nivel de apertura, el cual mantiene una relación significativa no sólo en la generación de patentes, sino también la solicitud y publicación de artículos científicos, siendo este último el que percibe un mayor impacto 2.4 por ciento por cada aumento del grado de apertura comercial de 1 por ciento.

Además, el pago de licencias es una variable que tiene impactos múltiples significativos, lo mismo que las importaciones. Lo que refuerza la premisa que establece que el rol de la fuerza global y especialmente las importaciones/exportaciones de tecnología son esenciales para la dinámica de la capacidad tecnológica en países en vías de desarrollo.

${ }^{16}$ Manifestada principalmente en patentes otorgadas, solicitadas, publicaciones y PIB per cápita. 


\section{III.3.3. Interpretaciones Modelo B}

Paralelamente, de acuerdo con las estimaciones del modelo B, se remarcan también los impactos detectados de los coeficientes que resultaron estadísticamente significativos (véase cuadro 9 y figura 3 ).

\section{Cuadro 9. Resultados Modelo B}

\begin{tabular}{|c|c|c|c|c|c|c|c|c|c|c|}
\hline \multirow[t]{3}{*}{ Dimensión } & \multirow{3}{*}{\multicolumn{2}{|c|}{$\frac{\text { Modelo B }}{\text { Variables }}$}} & \multicolumn{8}{|c|}{ LOS RESULTADOS LOGRADOS } \\
\hline & & & \multicolumn{2}{|c|}{$\begin{aligned} \text { Y1 = } & \text { PATENTES OTOR- } \\
& \text { GADAS }(\text { Ln) }\end{aligned}$} & \multicolumn{2}{|c|}{$\begin{array}{l}\text { Y2= SOLICITUDES } \\
\text { DE PATENTE (Ln) }\end{array}$} & \multicolumn{2}{|c|}{$\begin{aligned} Y 3= & \text { PUBLICACIONES } \\
& \text { CIENT }(L n)\end{aligned}$} & \multicolumn{2}{|c|}{$\begin{array}{c}\text { Y4= PIB PER CÁPI- } \\
\text { TA (Ln) }\end{array}$} \\
\hline & & & $\beta$ & SE & $\beta$ & SE & $\beta$ & SE & $\beta$ & SE \\
\hline \multirow{8}{*}{$\mathrm{BD}$} & \multicolumn{10}{|c|}{ Capacidades tecnológicas de infraestructura } \\
\hline & $\mathrm{X} 1$ & $\begin{array}{c}\text { Stock de patentes } \\
\text { (Ln) }\end{array}$ & $(2.1239)^{* * * *}$ & 0.3804 & $0.934^{* * * *}$ & 0.186 & $0.2595^{* * * *}$ & 0.035 & 0.024 & 0.055 \\
\hline & X3 & Telefono (Ln) & 0.3601 & 0.283 & $(-0.281)^{*}$ & 0.167 & $0.599 * * * *$ & 0.130 & $(-0.005)$ & 0.040 \\
\hline & $\mathrm{X} 4$ & Celular (Ln) & 0.1549 & 0.1442 & -0.073 & 0.068 & $(-.2646)^{* * * *}$ & 0.050 & $0.073^{* * * *}$ & 0.018 \\
\hline & \multicolumn{10}{|c|}{ Capacidades de absorción (acervo de recursos humanos) } \\
\hline & $\mathrm{X} 7$ & $\begin{array}{l}\text { Investigadores } \\
\text { I+D (Ln) }\end{array}$ & $(0.4324)^{* * * *}$ & 0.1257 & -0.117 & 0.082 & $0.660^{* * * *}$ & 0.059 & $(-0.014)$ & 0.011 \\
\hline & & & & "Inse & rción comercial & internac & ional & & & \\
\hline & $\mathrm{X} 10$ & Apertura (Ln) & $(2.9060)^{* * * *}$ & 0.7003 & $0.929 * * *$ & 0.329 & $0.460 * * * *$ & 0.1221 & 0.0722 & 0.0677 \\
\hline \multirow{4}{*}{$E R$} & \multicolumn{10}{|c|}{ Inputs de absorción } \\
\hline & $\mathrm{X} 13$ & $\begin{array}{l}\text { Gasto en educa- } \\
\text { ción } \% \text { PIB }\end{array}$ & $(-.2993)^{* * * *}$ & 0.0621 & 0.037 & 0.039 & $(-.1543)^{* * * *}$ & 0.0358 & $(-.015)^{* * *}$ & 0.005 \\
\hline & \multicolumn{10}{|c|}{ Inputs Tecnológicos (adquisición de conocimiento externo) } \\
\hline & $\mathrm{X} 11$ & IED (Ln) & $(0.1441)^{* * *}$ & 0.0508 & $0.067^{*}$ & 0.037 & $0.264^{* * * *}$ & 0.042 & 0.002 & 0.004 \\
\hline \multirow{11}{*}{ NOTAS } & \multicolumn{2}{|c|}{ Constante } & 0 & omited & $(-8.470)^{* * * *}$ & 1.474 & $(-5.174)^{* * * *}$ & 0.658 & $8.677^{* * * *}$ & 0.4734 \\
\hline & \multicolumn{2}{|c|}{ Observaciones } & \multicolumn{2}{|l|}{144} & \multicolumn{2}{|l|}{157} & \multicolumn{2}{|l|}{167} & \multicolumn{2}{|c|}{167} \\
\hline & \multicolumn{2}{|c|}{ Núm de países } & \multicolumn{2}{|l|}{13} & \multicolumn{2}{|l|}{13} & \multicolumn{2}{|l|}{ NA } & \multicolumn{2}{|l|}{13} \\
\hline & \multicolumn{2}{|c|}{ R cuadrado } & \multicolumn{2}{|c|}{0.8893} & \multicolumn{2}{|c|}{0.9044} & \multicolumn{2}{|c|}{0.8846} & \multicolumn{2}{|c|}{0.9993} \\
\hline & \multicolumn{2}{|c|}{$\begin{array}{l}\text { Test de significancia } \\
\text { conjunta }\end{array}$} & 0.0000 & & 0.0000 & & 0.000 & & 0.000 & \\
\hline & FIV pr & omedio & 2.00 & & 2.09 & & 2.22 & & 2.22 & \\
\hline & $\begin{array}{l}\text { Breus } \\
\text { gragia }\end{array}$ & $\begin{array}{l}\text { ch and Pagan La- } \\
\text { an MTRE }\end{array}$ & 0.0000 & & 0.1681 & & 0.000 & & 0.000 & \\
\hline & Hausn & man test & 0.0000 & & 0.0000 & & 0.585 & & 0.001 & \\
\hline & Efecto & s fijos de paises & Significati & ivos & Significati & vos & No signific & ativos & Significa & tivos \\
\hline & Testp & arm i.year & 0.0000 & & 0.2420 & & 0.215 & & 0.000 & \\
\hline & Efecto & s fijos de tiempo & Significati & ivos & No significa & ativos & No signific & ativos & Significa & tivos \\
\hline
\end{tabular}




\begin{tabular}{|l|l|c|c|c|c|}
\hline $\begin{array}{l}\text { Wooldridge test Autoco- } \\
\text { rrelation }\end{array}$ & 0.0177 & 0.2576 & NA & 0.0000 \\
\cline { 2 - 5 } & Modified Wald test het & 0.0000 & 0.0000 & NA & 0.0000 \\
\cline { 2 - 5 } & Modelo Óptimo Final & PCSE_corregido & FGLS_corregido & MCO & PCSE_corregido \\
\hline
\end{tabular}

NOTAS ADICIONALES:

Abreviaturas: $\mathrm{BD}=$ Base Disponible; $\mathrm{ER}=$ Esfuerzos Realizados. $\mathrm{MCO}=$ Mínimos Cuadrados Ordinarios. PCSE $=$ Panel Corrected Standard Errors. FGLS= Feasible Generalizad Least Squares $.(\mathrm{Ln})=$ Logaritmo Natural. NA = No Aplica. SE= Error Estándar. FIV promedio = Factor de Inflación de la Varianza (cuando el valor de FIV es mayor a 10, se puede sospechar de problemas de multicolinealidad).

Significancia del: ${ }^{*} p=0.10 \quad * * p \leq 0.05 \quad * * * p \leq 0.01 \quad * * * * p \leq 0.001$

Fuente: Elaboración propia con base en resultados procesados en (Stata 12).

Figura 3. Elasticidades de los factores significativos del Modelo $B$

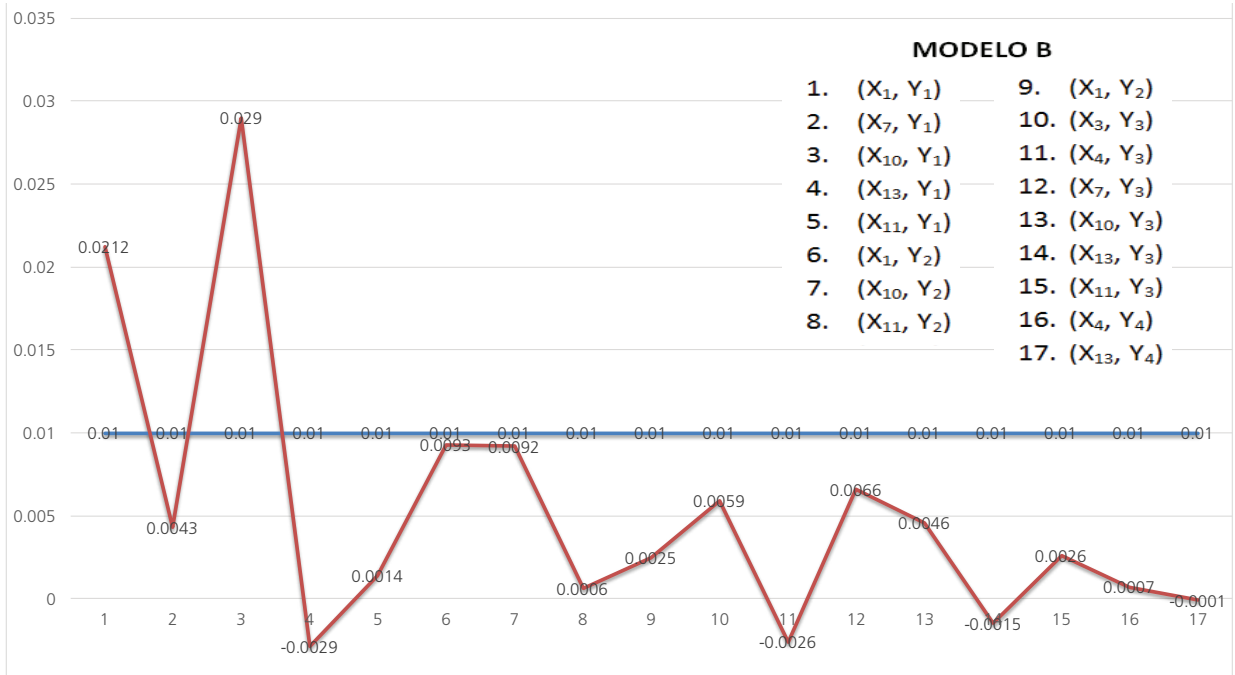

$-0.005-1 \% \times k i t-\Delta \% Y$

Fuente: Elaboración propia con base en resultados.

- Si el stock de patentes se incrementa en 1 por ciento, se espera que las patentes otorgadas aumenten 2.12 por ciento, las solicitudes de patentes se incrementen en 0.93 por ciento y las publicaciones científicas lo hagan en 0.25 por ciento.

El hallazgo anterior comulga con lo predicho por la teoría de desarrollo endógeno, ya que los resultados empíricos muestran a la variable "stock de patentes" como un factor determinante y significativo de la capacidad tecnológica en Latinoamérica, contrario a los hallazgos (Chinaprayoon, 2007), esta variable resultó insignificante dentro de su muestra de países desarrollados. Esto nos hace pensar 
que en el contexto latinoamericano, la dependencia de la trayectoria tecnológica, es decir de la acumulación de conocimientos, es crucial para entender la dinámica de la capacidad tecnológica de dicha región.

- Si las líneas telefónicas se incrementan en 1 por ciento, se espera que las solicitudes de patente tengan un decremento de 0.28 por ciento y las publicaciones científicas tengan un aumento de 0.59 por ciento.

- Si los usuarios de celular incrementan en 1 por ciento, se espera que las publicaciones científicas tengan un decremento del 0.26 por ciento y que el PIB per cápita tenga un aumento de .073 por ciento.

El proxy de la difusión de las viejas tecnologías, tiene un impacto positivo en el aumento de las publicaciones científicas, mientras que el proxy de la difusión de las nuevas tecnologías tiene externalidades negativas en estas últimas.

- Si el número de investigadores se incrementa en 1 por ciento, se espera que las patentes otorgadas aumenten en 0.43 por ciento y las publicaciones científicas en 0.66 por ciento.

El número de investigadores es otro de los factores que repercute en buena medida al incremento de la capacidad tecnológica, los países latinoamericanos tendrán que pugnar por incrementar el gremio. La necesidad de darle seguimiento a las estadísticas en este rubro es crucial para entender el comportamiento tecnológico actual y futuro.

- 1 por ciento de incremento en el gasto en educación, está ligado al decremento del .29 por ciento de patentes otorgadas, .15 por ciento de publicaciones científicas y .015 por ciento del PIB per cápita.

Se asume que el gasto en educación tiene sus impactos directos en la capacidad de absorción y no en la capacidad tecnológica.

- Si la Inversión Extrajera Directa (IED) se incrementa en 1 por ciento, se espera que las patentes otorgadas aumenten 0.14 por ciento y las solicitudes de patente lo hagan en .067 por ciento, las publicaciones científicas en 0.26 por ciento y el PIB per cápita en 0.002 por ciento.

La inversión extranjera directa (en el modelo B) y los grados de apertura (en el modelo A) implican el grado de conexión de un país a la comunidad internacio- 
nal. Puntualmente el nivel de inversión extranjera directa se emplea para capturar el grado de compromiso de un país a las capitales y tecnologías extranjeras. Los resultados de las estimaciones sugieren que entre más los países latinoamericanos se abren al comercio internacional, más se incrementa o consolida la capacidad tecnológica de la región.

En resumen, la importación de tecnología y la difusión tecnológica internacionales han demostrado ser elementos esenciales de la base disponible.

Estos dos indicadores (IED y apertura) implican la sólida relación entre las fuerzas del mercado internacional y el sector de la ciencia y la tecnología en los países en desarrollo.

En resumen, los determinantes de la capacidad tecnológica en Latinoamérica (bajo la base del modelo B) son principalmente el stock de patentes y el número de investigadores. Además que la Inversión Extrajera Directa (IED) es una variable que tiene impactos múltiples-significativos.

\section{CONTEXTUALIZACIÓN DE RESULTADOS: IMPLICACIONES EN EL ESCENARIO LATINOAMERICANO}

La experiencia de América Latina en relación con el diseño e implementación de políticas de CTI tiene sus origenes en los años cincuenta. Desde entonces, para fomentar las capacidades tecno-cientificas de la región se han promovido diferentes experimentos basados en paradigmas de política alternativos ${ }^{17}$ (Crespi y Dutrénit, 2013). Adicionalmente, tal como apuntan Morales, Ortiz y Arias (2012), los países en desarrollo, sobre todo los latinoamericanos, adoptaron el fenómeno del fortalecimiento de la ciencia, la tecnología y la innovación pero con una década de retraso o un poco más, comparado con los países de mayor desarrollo. De ahí pues la existencia y persistencia de una brecha tecnológica ${ }^{18}$ pronunciada entre ambos.

${ }^{17}$ Inicialmente se adoptó la política de Industrialización por Sustitución de Importaciones (ISI), nutrida de tecnología transferida en forma incorporada a las grandes inversiones de capital, sin que se prestara suficiente atención a las fases de adaptación a las condiciones de mercado, aprendizaje y todas aquellas que hoy se engloban en el concepto de trayectoria tecnológica de las firmas. El resultado fue una baja capacidad tecnológica del sector productivo de los países latinoamericanos, escasa demanda de conocimientos tecnológicos generados localmente y, por lo tanto, sistemas científicos escasamente vinculados con los procesos económicos y sociales. Al cabo de algunas décadas, el modelo de ISI fracasó en resolver el problema y, en algunos aspectos, hasta lo agravó, pese a haber alcanzado cierto éxito en impulsar el crecimiento de la industria de manufacturas en muchos países de la región (Albornoz, 2001).

${ }^{18}$ Existe, de hecho, una nueva "brecha tecnológica" en la globalización que divide las economías según su capacidad para la generación, asimilación y difusión del conocimiento. Esta 
Para Albornoz (2001), la usanza de América Latina en adoptar la política científica y tecnológica como instrumento de desarrollo, pese a ciertos logros en el plano académico, no puede ser considerada como un éxito. La crítica al modelo preexistente fue enfocada desde distintos ángulos. Desde uno de ellos se destacó el carácter marginal de la ciencia en la región, vinculándola con la dependencia de los centros de poder mundial. Desde esta perspectiva crítica se señalaba que la producción científica tenía más relación con las necesidades internas del grupo social que las generaba, que con los requerimientos propios del desarrollo del país dependiente y por lo tanto, se podía catalogar al sistema científico de los países latinoamericanos como exogenerado y endodirigido.

Crespi y Maffioli (2013), apuntan que desde comienzo de los años noventa, en varios paises de América Latina, se aprecia un crecimiento sistemático de los programas ${ }^{19}$ públicos dedicados a fomentar la innovación y la modernización tecnológica de las empresas. Sin embargo, en promedio, los países de América Latina tienen un desempeño menor en términos de productividad en comparación con otros, por ejemplo de la OCDE. De ahí que los resultados empíricos de Chinaprayoon (2007) para países en desarrollo mantenga divergencias con los hallazgos dentro del contexto latinoamericano, foco de este documento.

En la actualidad, se reconoce que algunos países de la región (por ejemplo, Argentina, Brasil, Chile y México) han comenzado a evolucionar hacia el desarro1lo de un perfil tecnológico más próximo al de las economías avanzadas. Estos países tienen una colección de instrumentos de política, así como recursos del sector público y privado que aún no están disponibles para otros países latinoamericanos. Se vislumbra que en general los países de esta región, se enfrentan a desafíos importantes en términos de la capacidad institucional, tales como: a) la necesidad de mantener las políticas a largo plazo, $b$ ) la necesidad de fortalecer la capacidad institucional para formular, supervisar y evaluar las políticas de innovación. c) la necesidad de desarrollar infraestructura de información para monitorear las políticas y los programas de CTI (Navarro, Benavente, y Crespi, 2016).

\footnotetext{
capacidad tiene que ver con el tipo de sociedad, con sus posibilidades de especialización para la competencia internacional y con la flexibilidad de sus ordenamientos normativos (Piñón, 2010).

${ }^{19}$ Varios países de la región de América Latina han estado experimentando con políticas de incentivos fiscales para apoyo a la innovación empresarial desde principios de los noventa, en muchos casos con el apoyo técnico y financiero del Banco Interamericano de Desarrollo (BID). A diferencia de los países de la OCDE, el marco de apoyo a la innovación empresarial en América Latina está claramente sesgado hacia trasferencias directas al sector privado. Sólo unos pocos países han comenzado más recientemente a experimentar con incentivos fiscales (Crespi y Maffioli, 2013).
} 
Bajo el contexto anterior, las investigaciones en torno a las capacidades tecnológicas y de innovación se vuelven pertinentes y justifican los esfuerzos encaminados a proveer evidencia empírica del comportamiento tecnológico de esta región, a sabiendas que los hallazgos tienden a fundamentar políticas regionales acordes con las realidades y necesidades del territorio estudiado.

\section{IV.1. Implicaciones de los determinantes de la capacidad tecnológica en Latinoamérica}

Consecuentemente con lo anterior expuesto, se esbozan las implicaciones de los resultados encontrados, aludiendo pues a las connotaciones que es posible asignarles a los factores determinantes de la capacidad tecnológica en el contexto latinoamericano.

\section{IV.2. Impactos de la apertura comercial}

El grado de apertura externa ${ }^{20}$ es el indicador más usual para medir la proyección internacional de las economías, se le conoce también como el grado de apertura externa o apertura comercial de las economías (OCDE , 2015).

La evidencia empírica de este trabajo muestra que tanto en el modelo A como para el B, la apertura comercial no sólo es estadísticamente significativa a la hora de evaluar la capacidad tecnológica en Latinoamérica, sino que además muestra elasticidad, es decir que ante cambios del 1 por ciento en dicha variable, se esperan incrementos de mayor proporción tanto en las patentes solicitadas como en las otorgadas, al tiempo de fungir como un factor importante en la generación de publicaciones científicas.

En ese sentido, es importante mencionar entonces que según López (2010), una economía estará más presente en los mercados internacionales cuanto mayor sea su grado de apertura, esto es, cuanto mayor sea la participación de la suma de las exportaciones más las importaciones en la producción nacional.

${ }^{20}$ Este indicador se define como el porcentaje que suponen los flujos del comercio exterior, exportaciones (X) e importaciones (M) en el total del PIB. Su debilidad radica en que otorga el mismo tratamiento a las importaciones y a las exportaciones, por lo que dos economías pueden presentar el mismo grado de apertura con flujos comerciales cuya magnitud de cada uno de sus direcciones sean muy dispares (López, 2010). 
En otras palabras, la dinámica de exportación e importación juega un rol determinante en la generación de la capacidad tecnológica de la región, por lo que futuros estudios deberán considerar analizar la composición tanto de importaciones como de exportaciones en cada país a efectos de identificar las peculiaridades de este comportamiento en cada contexto.

Al respecto Sachs y Vial (2012), apuntan que el incremento y diversificación de las exportaciones son sólo algunos de los resultados del libre comercio. En términos generales, el aumento del comercio ha sido un buen indicador de transformaciones estructurales ${ }^{21} \mathrm{y}$ del nivel de integración -comercial- a la economía global. No obstante, es bien sabido que la liberalización comercial es también uno de los aspectos más controvertidos de la globalización; muchos consideran que los supuestos costes superarán a los beneficios que se pretende conseguir: mayor eficiencia y aumento del crecimiento. En ese sentido, la liberalización comercial expone a los países a más riesgos (reducción salarial, aumento de desempleo, pérdida de la soberanía nacional) y los países en vías de desarrollo (y sus trabajadores) están menos preparados para asumir dichos apuros (Stiglitz, 2006). Por lo que en este punto habrá que clarificar que el aumento del grado de apertura comercial en aras de mayores niveles de capacidad tecnológica tendrá que ser sopesado a fin de no acarrear mayores desigualdades.

Tal como enfatiza el Banco Mundial (2015), es necesario considerar que lo importante no es "qué es lo que se produce (exporta) sino el cómo" apuntando a la importancia del desarrollo de "redes del conocimiento" que favorezcan la innovación y adopción de tecnologías. En ese sentido, la exportación de importaciones tecnológicas ${ }^{22}$ es un tema problemático que tendrá que ser analizado país por país,

${ }^{21}$ En dónde se encuentra que únicamente en Jamaica y Chile se puede encontrar una relación directa entre las políticas para eliminar las barreras al comercio, y un incremento continuo de la importancia del comercio en el PIB. Costa Rica, República Dominicana y México han tenido éxito en atraer inversión extranjera a través de incentivos específicos, como las Zonas Francas en República Dominicana, o el Tratado de Libre Comercio de América del Norte (TLCAN). En los tres casos, el acceso al mercado estadounidense les ha permitido la integración de las plantas de producción de estos países dentro de la cadena de producción de los Estados Unidos brindándoles la oportunidad de incrementar, tanto como la importación de partes y materiales, como la exportación de productos semiterminados o terminados. Otros países con una base fuerte de productos industriales, y con una presencia importante de empresas multinacionales en el país, como Brasil o Argentina, han fracasado a la hora de convertir esta ventaja inicial en un incremento en los flujos de comercio (Sachs y Vial, 2012).

${ }^{22}$ Exportar importaciones es problemático porque muestra: 1) la poca competitividad que tienen los bienes nacionales (que han tenido que ser substituidos por internacionales) y/o, 2) la alta dependencia que se tiene del precio del dólar (que al subir incrementa proporcionalmente 
evaluando también sus causas y consecuencias. Esto, dado que en los países en vías de desarrollo se evidencia una tendencia hacia la importación de tecnologías externas, como un factor importante en las capacidades de innovación vistas desde una perspectiva netamente tecnológica (Morales, Ortiz, y Arias 2012).

\section{IV.3. Impactos del stock de patentes}

La teoría de crecimiento endógeno predice que los impactos del stock de patentes son determinantes para la generación de capacidades tecnológicas, los hallazgos obtenidos mediante este estudio refuerzan dicha premisa, encontrando que dicho factor dentro del modelo de regresión $\mathrm{B}$, tiene impactos significativos en las variables explicadas (patentes otorgadas, solicitudes de patente y publicaciones).

Lo anterior tiene una interpretación más profunda que la estadística, si se asume que la variable "stock de patentes" tiene per se una connotación mucho más amplia. En primer lugar habrá que ubicarla dentro de los elementos más importantes de lo que la CEPAL (2007), denomina "la base disponible" que en otras palabras alude a los recursos humanos y la infraestructura, que revelan la "calidad" del entorno, consecuentemente, se puede considerar a dicha variable no sólo como un indicador de la acumulación de conocimiento de una nación, sino además como un semáforo de la situación contextual de la región.

Bajo tal escenario, el pensamiento CEPALino, ha aducido el concepto de heterogeneidad estructural, interpretada como resultado de las condiciones históricas heredadas y de la evolución natural del mercado de trabajo y por consecuencia de las trayectorias tecnológicas, indicando que el acento neoschumpeteriano en la formación y acumulación de conocimiento mediante el proceso de aprendizaje de las empresas y naciones, el efecto de las decisiones del pasado sobre las del presente (path-depency) y la modificación de los paradigmas tecno económicos que enriquecen el enfoque histórico-estructural aplicado por la CEPAL, es un elemento esencial para comprender las trasformaciones de las estructuras productivas en condiciones de subdesarrollo y heterogeneidad estructural (CEPAL, 2015).

En ese sentido, Amarante, Galvan, y Mancero (2016), encuentran que en América Latina la lectura de los datos confirma también que uno de los rasgos predominantes en la región en materia de ciencia y tecnología es el de la heterogeneidad. La pluralidad de situaciones impone la aplicación de instrumentos y modelos diferenciados en las políticas de ciencia y tecnología de los países latinoamericanos.

el porcentaje de gasto que las empresas exportadoras destinan a comprar bienes extranjeros comparados con nacionales), (Ríos 2015). 
Sin embargo, este rasgo se contrapone con la tendencia de los programas de cooperación internacional a prestar escasa consideración a las diferencias y proponer recetas semejantes. Hay un contraste entre la heterogeneidad de las situaciones nacionales y la homogeneidad de las acciones emprendidas por la cooperación internacional en ciencia y tecnología. En donde la diversidad de la región abre oportunidades para el ejercicio de la cooperación horizontal, ya que los países de mayor tamaño relativo tienen la oportunidad de mostrarse solidarios con relación a los más pequeños de América Latina.

En comunión con lo anterior, Botargaray y Gras (2013) añaden que lo que sí parece claro es que la diversidad de contextos y de trayectorias, requieren de nuevos marcos analíticos y políticas en Сті diversas y plurales. Sin embargo, a pesar de las notables diferencias en las trayectorias y en los diversos tipos de problemas que enfrentan paises desarrollados y en desarrollo, el conjunto de instrumentos y política orientada a impulsar la CTI han sido relativamente uniformes. Si bien el diseño del conjunto de políticas debería responder a la naturaleza específica y al tipo de problemas, necesidades, demandas y capacidades locales, la práctica habitual ha sido la intersección con instrumentos similares, desestimando su idoniedad y capacidad de respuesta a las dinámicas locales.

Para Hernández (2016), la desigualdad del ingreso en América Latina es una de las más altas del mundo y tiene una doble razón de ser. En buena medida se debe a las diferencias en cuanto a capacidades (pieza de nuestro estudio). Sin embargo, la parte más importante de la desigualdad del ingreso se debe a que la población del continente participa en los mercados con desigualdades iniciales, históricas y abismales: desigualdades de coberturas básicas, de acceso a mercados, de capital humano, de información, de acceso efectivo a derechos y, sobre todo, de poder.

No es pues de sorprender que, bajo los resultados empíricos del presente estudio, el factor denominado "stock de patentes" haya sido identificado como esencial en la generación de capacidades tecnológicas para la región. Lo que sí es de sorprender es que ante la heterogeneidad de la región se continúen con las prescripciones genéricas, por tanto, habrá entonces que considerar las recomendaciones citadas por los expertos del tema en cuanto a la aplicación de instrumentos y modelos diferenciados en las políticas de ciencia y tecnología de los países latinoamericanos.

\section{IV.4. Impactos del gasto $I+D$}

La evidencia empírica de este trabajo sugiere que dentro del modelo A, los gastos de I+D (como porcentaje del PIB) no sólo son estadísticamente significativos a la hora de evaluar la capacidad tecnológica en Latinoamérica, sino que además muestra elasti- 
cidad, es decir, que ante cambios de 1 por ciento en dicha variable, se esperan incrementos de mayor proporción principalmente en la obtención de patentes internacionales. La estimación puntual recalca que ante aumentos de 1 por ciento de la variable en cuestión, se esperarían aumentos de las patentes otorgadas en 1.46 por ciento.

El hallazgo anterior sorprende en cuanto a la proporción descubierta, ya que anteriormente, de acuerdo con las estimaciones de Chinaprayoon (2007), 1 por ciento de incremento en el gasto en $\mathrm{I}+\mathrm{D}$, lleva a un aumento de .59 por ciento en las patentes otorgadas en países en vías de desarrollo de todo el mundo. En ese sentido el BID (2014), argumenta que muchas veces se cree que una de las pocas ventajas de un país en desarrollo es que puede sencillamente sacar provecho de las inversiones en innovación de los países desarrollados y en este caso, las empresas provenientes de dichos países podrían acaparar o incrementar significativamente su presupuesto de $\mathrm{I}+\mathrm{D}$ gracias a los recursos públicos destinados a tal fin y dado que el retorno de una determinada tecnología depende del contexto en que se utiliza. La disponibilidad de insumos complementarios clave, como el capital humano, las instituciones y los recursos naturales, propios de la región estudiada pudiesen estar influyendo a favor (unas más que otras) dentro del fenómeno detectado.

$\mathrm{El}$ hecho que el gasto en $\mathrm{I}+\mathrm{D}$, no resulte el principal factor determinante para la generación de capacidades tecnológicas pudiese parecer poco usual, sin embargo, las teorías recientes sobre la base de la economía evolutiva sugieren que los resultados de la innovación y otros logros tecnológicos no son simplemente el resultado de un aumento de ciertos insumos, sino, son más bien producto de actividades complejas e interrelacionadas de sistema productivo. En el caso latinoamericano, la apertura comercial y el stock de patentes se muestran con mayor impacto por encima del "gasto en I+D".

Paralelamente a nuestros hallazgos, BID (2014), encuentra que la tasa de retorno social para la inversión en I+D no sólo es sistemáticamente más alta en América Latina y el Caribe que en la OCDE (56 por ciento vs. 32 por ciento en 2007) sino también que ha seguido una trayectoria divergente a lo largo del tiempo. Mientras que los retornos sociales han disminuido en la OCDE (debido fundamentalmente al menor retorno de la trasferencia tecnológica a medida que estos países se han acercado a la frontera de producción), en América Latina y el Caribe el retorno social a la $\mathrm{I}+\mathrm{D}$ ha tendido a aumentar (sobre todo debido al mayor espacio para la trasferencia tecnológica).

Adicionalmente, Navarro, Benavente, y Crespi (2016), señalan que los esfuerzos para mejorar la inversión en $\mathrm{I}+\mathrm{D}$ en América Latina se concentraron en un puñado de países. La financiación de la I+D sigue estando muy concentrada en las instituciones públicas (agencias gubernamentales y universidades), la mayor parte de esta inversión pública se gasta en las instituciones públicas (organismos 
públicos de investigación y laboratorios), ya que los programas públicos destinados a subvencionar los negocios y la innovación del sector privado suelen ser muy pequeños. Sin embargo, los acontecimientos recientes en algunos países de la región sugieren que la tendencia de invertir insuficientemente en CTI está comenzando a invertirse.

Para Crespi (2014), hay a todas luces un déficit importante en inversión en I+D en la región, sobre todo en el sector privado. Aun teniendo en cuenta la adquisición de tecnología incorporada en maquinarias y equipos, donde el déficit es menor, la falta de inversiones en I+D y el bajo capital humano en la región socava gravemente la efectividad con que se pueden usar las tecnologías incorporadas. Este déficit de inversiones en innovación ha impedido a la región converger con el resto del mundo en lo que respecta a la productividad.

Recientemente, Mongue y Tacsir (2013), encuentran que la mayoría de las actividades productivas de los mercados de América Latina (a nivel industrial o de servicios) no tienen relación con los esfuerzos de innovación, es decir, las señales de ganancias relativas a corto plazo parecen estar disociadas de la innovación.

En conclusión, tal como argumenta CEPAL (2007), estos países deberán aumentar significativamente los esfuerzos destinados a una sustancial mejora de la base disponible, sobre todo en lo que respecta a un salto significativo en las capacidades de absorción y una mayor y más equilibrada disponibilidad de infraestructura.

\section{IV.5. Impactos en el número de investigadores}

Los hallazgos detectados sugieren que dentro del modelo B, el número de investigadores es un factor estadísticamente significativo a la hora de evaluar la capacidad tecnológica en Latinoamérica. La estimación puntual recalca que ante aumentos de 1 por ciento de la variable en cuestión, se esperarían aumentos de las patentes otorgadas en .43 por ciento y .60 por ciento de las publicaciones científicas. Sin embargo, dicha variable muestra inelasticidad, ya que las variables explicadas (de capacidad tecnológica) varían en menor proporción que la de la variable "número de investigadores". En otras palabras, se necesita duplicar el esfuerzo porcentual del factor "número de investigadores" para conseguir aumentos proporcionalmente similares en la generación y solicitud de patentes internacionales y publicaciones científicas.

En ese sentido, SciDev (2015), señala que contar científicos puede sonar cosa fácil, pero las incertidumbres asociadas sugieren que es un asunto enredado. Una fuente bien reputada, la Red de Indicadores de Ciencia y Tecnología (RICYT), sostenida por la Organización de Estados Iberoamericanos, acusa huecos 
en sus bases de datos y en algunos países de la región no existen estadísticas actualizadas en materia de ciencia y tecnología y no se cuenta con información certera sobre el número de científicos. Por lo tanto, hace falta una noción más profunda de "medir la ciencia" no sólo con el conteo de científicos sino también evaluando las condiciones de investigación. Más aún, si bien podemos acordar que Latinoamérica necesita más de 250 mil científicos, también necesita preguntarse para qué los quiere. Que sean muchos o pocos dependerá de cuánta importancia tiene la ciencia en la estrategia de desarrollo y qué tan compatible con la ciencia sea la cultura dominante.

Adicionalmente, según Navarro, Benavente, y Crespi (2016), la tasa de crecimiento de las publicaciones de América Latina se triplicó entre finales de 1990 y mediados de la década de los 2010, por lo tanto, se ha podido superar a algunas otras regiones y consecuentemente se ha podido reducir la brecha -al menos en cuanto a publicaciones-. La naturaleza de la investigación en las economías latinoamericanas también es diferente de la de los países de la OCDE. En ese sentido resulta útil cuestionarse no sólo cuántos investigadores hay en la región sino también qué investigan, ya que la parte de los investigadores que trabajan en los campos de ingeniería y tecnología (como porcentaje del total de investigadores) está entre el 10 y el 30 por ciento (RICYT, 2014), mientras que en países como Singapur, Japón y Corea del Sur esta cifra es del 60 por ciento.

\section{IV.6. Las variables con efectos inelásticos}

Es conveniente mencionar aquellos factores que resultaron estadísticamente significativos pero inelásticos. Bajo el modelo de regresión A, la variable "pago de licencias" mostró impactos positivos y significativos en las solicitudes de patente, las publicaciones científicas y el PIB per cápita. Paralelamente, la variable de "importaciones" evidenció efectos significativos en la generación de patentes internacionales y ligeramente en el incremento del PIB per cápita. Mientras que en el modelo de regresión $\mathrm{B}$, la variable IED mostro impactos positivos y significativos en generación de patentes internacionales, las solicitudes de patente, las publicaciones científicas y el PIB per cápita. Lo anterior, según la CEPAL (2015) puede ser explicado dado que la inversión extranjera directa es una fuente importante de obtención de nuevas tecnologías para los países en desarrollo, sin embargo, su importancia en América Latina parece ser menor que en otras regiones, probablemente debido a la excesiva orientación hacia el abastecimiento de mercados internos de IED que llega a esta región, con la excepción reciente de México, República Dominicana y Costa Rica. 
En otras palabras, se necesita un esfuerzo porcentual mayor del de estas variables inelásticas para conseguir aumentos proporcionalmente similares en el incremento de las capacidades tecnológicas latinoamericanas.

Cabe mencionar entonces, que en el contexto estudiado, la atracción de IED ha cumplido un rol preponderante en las nuevas políticas de desarrollo productivo. $\mathrm{Si}$ bien las políticas de IED han sido bien acogidas en general, algunos países se han esforzado por atraer IED hacia sectores específicos, tecnológicamente avanzados, y para emprender las acciones requeridas a fin de asegurar el éxito de dicha iniciativa (BID, 2014).

En algunos países latinoamericanos, ${ }^{23}$ se considera a la atracción de la IED como una opción estratégica para mantener el crecimiento, promover el cambio estructural y crear mejores puestos de trabajo. Sin embargo es necesario que la IED sea más óptima y que la región vaya cambiando gradualmente hacia un enfoque de política más selectiva de la IED orientándose a ciertos sectores intensivos en conocimiento, mientras que algunas empresas globales se han movido recientemente hacia actividades crecientemente sofisticadas. En general, las experiencias exitosas de todo el mundo muestran que la IED intensiva en conocimiento es más sensible a la disponibilidad de las habilidades y las capacidades de investigación (incluyendo los laboratorios de $\mathrm{I}+\mathrm{D}$, plataformas de innovación, grupos de empresas y centros de investigación) que fomenten un entorno innovador. Buenas prácticas en este campo demandan mayores niveles de integración y coordinación entre las políticas de promoción de la IED y las políticas complementarias claves en las áreas de innovación, educación e industria (Mongue y Tacsir, 2013).

Por otra parte, en lo que respecta a las importaciones, cabe mencionar que el depender de la tecnología importada no es necesariamente malo ${ }^{24}$ si se poseen suficientes capacidades de absorción y si ello conduce a procesos de aprendizaje. Sin embargo, para que esto suceda, se debe combinar la tecnología adquirida con esfuerzos en investigación y desarrollo y capital humano complementario. No es ninguna sorpresa que el proceso de innovación en América Latina se base en la

${ }^{23}$ En Costa Rica, por ejemplo el exitoso desempeño de la IED en el país fomentó cambios profundos en la especialización comercial, provocando una demanda de nuevas y mejores habilidades de la población y una mayor disponibilidad de capacidades empresariales y técnicas. De hecho, la movilidad de trabajadores desde las empresas globales a nacionales ha tenido un impacto positivo en la tasa de creación y supervivencia de empresas intensivas en conocimiento en el país.

${ }^{24}$ De acuerdo con CEPAL (2015), los países de América Latina actúan principalmente como receptores de la innovación del exterior, lo cual no es tan malo si se considera que Corea o Singapur, que actualmente pertenecen al grupo de los líderes innovadores (o por lo menos se encuentran en el límite para serlo), eran únicamente receptores de tecnología extranjera hace sólo una década. 
adopción y en la mejora incremental de tecnologias existentes, más que la inversión en I+D (BID, 2014). Sin embargo, según apunta Botella y Suarez (2012), para contribuir al estímulo de la financiación privada de la innovación en América Latina, habría que contar, en primer término, con las empresas multinacionales de la propia región (las llamadas multilatinas). Estas empresas, con importante peso en diversos sectores en el ámbito global, pueden canalizar el potencial innovador en los países de la región y reequilibrar el tradicional flujo de producción de la innovación, que se generaba principalmente en los países desarrollados, especialmente en la Unión Europea y Estados Unidos, para acabar implantándose en América Latina. En segundo lugar, habría que considerar el papel de las empresas internacionales que cuentan con una relevancia considerable en la región. Estas empresas pueden contribuir al impulso innovador a través de la transferencia tecnológica, la financiación y el apoyo a iniciativas innovadoras surgidas en los países latinoamericanos.

Por su parte, Stiglitz y Greenwald (2014), señalan que el aprendizaje en los países en vías de desarrollo ocurre a través de diversos canales como: la movilidad de las personas, formas de código abierto de propagación del conocimiento, bienes de inversión, imitación e ingeniería inversa (por ejemplo, a través de empresas multinacionales), inversión extranjera directa y adquisición de empresas con tecnología. Sin embargo, estos países deben considerar seriamente que unos derechos de propiedad intelectual más fuertes (y, sobre todo, los que están pobremente diseñados) tendrían un efecto en extremo adverso sobre el aprendizaje y la innovación en los países en vías de desarrollo.

\section{Conclusiones}

El objetivo del presente trabajo fue examinar los determinantes de la capacidad tecnológica en Latinoamérica. Para ello, se desarrolló un modelo econométrico utilizando la técnica de datos de panel con efectos fijos, en base al set de indicadores propuesto por CEPAL (2007) y Chinaprayoon (2007), y recopilando los datos de diversas fuentes públicas, utilizando una serie temporal de 14 años y una dimensión transversal de 17 países; esto con el fin de analizar el grado de impacto de las variables relativas a la capacidad tecnológica en América Latina. A partir de la estimación econométrica, se encuentra que la apertura comercial, el stock de patentes y el gasto en $\mathrm{I}+\mathrm{D}$, son los determinantes más influyentes de la capacidad tecnológica en dicho contexto. Las estimaciones puntuales concentradas en el cuadro 10, son hallazgos identificados por primera vez en Latinoamérica mediante este trabajo. Estudios previos habían analizado las capacidades tecnológicas en países desarrollados y altamente industrializados. 
114 ECONOMÍA TeOría Y PRÁCTICA • Nueva Época, número 48, enero-junio 2018

Cuadro 10. Hallazgos empíricos de la capacidad tecnológica en Latinoamérica

\begin{tabular}{|l|l|l|l|}
\hline \multicolumn{2}{|l|}{ Modelo A } & \multicolumn{2}{l|}{ Modelo B } \\
\hline$\Delta 1 \%$ Impactos significativos & $\Delta 1 \% X i$ & Impactos significativos \\
\hline 1) Apertura & $\begin{array}{l}0.41 \% \text { patentes } \\
2.40 \% \text { solicitudes de patentes } \\
0.52 \% \text { publicaciones }\end{array}$ & 1) Apertura & $\begin{array}{l}2.90 \% \text { patentes otorgadas } \\
0.92 \% \text { solicitudes de patente } \\
0.46 \% \text { publicaciones }\end{array}$ \\
\hline 2) Gasto en I+D & $1.46 \%$ patentes & 2) Stock de patentes & $\begin{array}{l}2.12 \% \text { patentes otorgadas } \\
0.93 \% \text { solicitudes de patente } \\
\end{array}$ \\
\hline 3) Líneas telefónicas & $1.04 \%$ patentes & & $0.25 \%$ publicaciones \\
\hline $\begin{array}{l}\text { Las variables: "pagos de licencias" e "importaciones" tienen } \\
\text { efectos múltiples-ligeros en las variables dependientes. }\end{array}$ & 3) Investigadores & $\begin{array}{l}0.43 \% \text { patentes otorgadas } \\
0.60 \% \text { publicaciones }\end{array}$ \\
\hline
\end{tabular}

Nota: la interpretación se da mientras todas las demás variables permanezcan constantes.

Fuente: Elaboración propia.

Posteriormente, derivado de la contextualización de los resultados, se logró no sólo interpretar las posibles causas de los hallazgos, sino además identificar áreas de oportunidad en materia de política de CTI para la región.

Todo indica que a fin de lograr mejores resultados en materia de capacidades tecnológicas y la reducción de la brecha con respecto de los países desarrollados, Latinoamérica deberá aumentar significativamente los esfuerzos realizados (gasto en $\mathrm{I}+\mathrm{D}$ ) a sabiendas que los resultados serán más redituables (en la generación de patentes internacionales) en comparación que otros países en desarrollado de la OCDE. Pero principalmente, la región deberá prestar atención al grado de apertura comercial y evaluar su balanza tecnológica, ya que en el contexto analizado, la dinámica de exportaciones e importaciones es un factor elemental para la generación de outputs tecnológicos. Finalmente se deduce que los factores que integran la base disponible son los que mayor impacto tienen sobre la acumulación y consolidación de capacidades tecnológicas, por lo tanto, será necesario reforzar las capacidades de absorción y una mayor y más equilibrada disponibilidad de infraestructura.

Además, las políticas públicas deberán velar por la producción directa de conocimiento en instituciones públicas (laboratorios e institutos de investigación de carácter público) y los incentivos fiscales para lograr una mayor cantidad de inversiones privadas en la generación de conocimientos (Crespi y Maffioli, 2013).

Para Morales, Ortiz, y Arias (2012), es evidente que en el caso de los países latinoamericanos se han evidenciado trasformaciones notables en los sistemas productivos durante las últimas dos décadas, tiempo durante el cual se han hecho esfuerzos de ordenamiento macroeconómico, diferentes reformas de mercado (apertura comercial, desregulación de los mercados y privatización de activos 
públicos), y políticas de reinserción internacional, que a pesar de no ser suficientes, marcan el camino hacia el mejoramiento de sus sistemas de innovación.

Se propone que en América Latina es preciso reorientar la modalidad productiva y exportadora hacia los bienes de mayor contenido tecnológico y, al mismo tiempo, agregar valor mediante cadenas sectoriales de mayor envergadura. Y a medida que los países incorporen nuevas tecnologías, deben ir aplicando políticas que fomenten la cooperación entre las empresas y las instituciones de investigación. Además, conforme se vaya logrando llegar a los estándares de calidad en educación y salud necesarios para promover el desarrollo, se debe ir aumentando la cantidad de recursos destinados a la investigación y la innovación (CEPAL, 2015).

Adicionalmente, Stiglitz y Greenwald (2014), advierten que la propiedad intelectual no es un fin en sí mismo. Las patentes, por tanto, no son signo de innovación, pero ciertamente sí son señal de la capacidad tecnológica que le subyace. Por lo tanto, principalmente, se debe promover la actividad intelectual creativa y la transferencia de tecnología hacia los países en vías de desarrollo. De tal suerte que la nueva agenda de desarrollo llame a determinar la forma cómo los distintos regímenes de propiedad intelectual afectan a los países en vías de desarrollo como es el caso de la región latinoamericana.

Se requerirá pues de capacidades científicas básicas para seguir el ritmo de las múltiples aplicaciones de la ciencia en los campos de gran importancia económica, como la energía, la protección del medio ambiente, el transporte, las telecomunicaciones y la agricultura. En este sentido, la I+D deben ser vistos como fuente de ideas originales y una fuente de la capacidad de absorción para buscar ideas y adaptar las condiciones tecnológicas existentes.

Los programas y políticas destinados a mejorar el rendimiento de la innovación deben ser personalizados para reflejar estas diferencias. Por lo tanto, los países que ya han construido una capacidad sustancial en la investigación científica pueden necesitar programas que hacen hincapié en el mantenimiento de esa capacidad y, sobre todo, los enfoques que se centran en la conexión de estas capacidades con el sector productivo (Navarro, Benavente, y Crespi, 2016).

Además, según Crespi (2014), las economías latinoamericanas que aún no tienen capacidad de investigación científica tendrán que hacer hincapié en la creación de políticas de ciencia básica y la innovación (por ejemplo, la incorporación, los procesos de selección basados en la competencia revisadas por pares para la investigación y los proyectos de innovación en firme) y la adquisición de una masa crítica mínima de recursos humanos de capital para la innovación. Desde un punto de vista institucional, los países con una tradición de ciencia y política de desarrollo productivo pueden tener que invertir principalmente en la coordinación y la coherencia global de sus entornos institucionales. Los países con pocos prece- 
dentes institucionales, es posible que deseen invertir en una amplia variedad de instrumentos que no son exigentes en términos de capacidad de ejecución, pero permitir un proceso de aprendizaje rápido, tanto en el sector público y privado.

Finalmente, se recalca que futuros estudios podrían utilizar alguno de los dos modelos de regresión (A o B) para generar indicadores sintéticos, además es pertinente que los analistas económicos puedan dar evidencia empírica de la experiencia específica de cada país latinoamericano a fin de conocer a profundidad las prácticas de Сті implementadas y sus efectos sociales, económicos y tecnológicos.

\section{DISCUSIONES Y REFLEXIONES}

Abordar el tema de las capacidades tecnológicas de manera empírica y el tópico de la tecnología y sus efectos puede resultar controversial en algún sentido. En el primer caso, los estudios econométricos tendrán siempre cierto grado de abstracción lo cual inevitablemente conduce a elegir ciertas variables y descartar otras (por ejemplo, ha sido ampliamente discutido el tema de la utilización de patentes ${ }^{25}$ como un indicador de innovación, sin embargo, como señalador de capacidad tecnológica, éste se vuelve más pertinente; porque, aunque no representa un éxito comercial puede ser considerado como un éxito tecnológico). Más aún, es importante resaltar que la realización de estudios empíricos relacionados con la capacidad tecnológica de los países en desarrollo no es tan basta, por lo que resulta por demás justificable encaminar investigaciones enfocadas a ampliar o complementar el entendimiento en cuanto al comportamiento de la capacidad tecnológica en dichos contextos. En el segundo caso, es decir cuando se aborda el tema tecnológico per se surgen convergencias y divergencias en cuanto a sus implicaciones sobre el desarrollo económico (desarrollo social, ambiental, económico e inclusivo). Por una parte, la postura optimista del elemento tecnológico argumenta por ejemplo que "el progreso tecnológico genera más trabajo que el que destruye" no obstante esto implica que el cambio tecnológico sea un factor crucial para el crecimiento desigual (Brynjolfsson y McAfee, 2013). Por lo que, en ese sentido, en futuras investigaciones podría cuestionarse la hipótesis en la que según Tedesco (2010), las sociedades que están utilizando más intensivamente la información y los conocimientos en sus actividades productivas, están aumentando significativamente la desigualdad social; donde la polarización social es resultado de un sistema institucional que no se hace responsable del destino de las personas. Y tal como argumenta Stiglitz (2012), es indudable que los cambios en la tecnología puedan dar lugar a una reducción de la demanda de mano de obra en algún sector, y el cambio en los sectores productivos

${ }^{25}$ Véase Archibugi (1998). 
es uno de los factores clave en el aumento de la desigualdad. Sin embargo, se evidencia también que las políticas tienen un gran efecto distributivo, en este caso, es evidente que en el contexto latinoamericano se requiere más bien de políticas diferentes que ayuden a disminuir la alta heterogeneidad estructural y tecnológica de la región. A efectos de mitigar lo que Albornoz (2001), denomina como el "efecto Mateo": dios le da más al que más tiene. En el entendido de que si la ciencia se ha convertido en una fuerza de producción, entonces reproduce la estructura social. Se convierte en un instrumento que hace más ricos a los ricos y más pobres a los pobres. Bajo este documento, estamos claros que el desarrollo para pocos y a cualquier precio, simplemente no es desarrollo.

En ese sentido Botella y Suárez (2012), señalan que las predicciones económicas apuntan a que el crecimiento se mantendrá robusto en la mayoría de los países en América Latina durante los próximos años. A pesar de ello, habrá que hacer distinción entre crecimiento y desarrollo, ya que de acuerdo con Botargaray y Gras (2013), es compartida la idea de que existe una relación fuerte y positiva entre CTI y crecimiento económico, sin embargo, cuando se piensa en CTI y desarrollo, dicha relación se desvanece, y su complejidad hace que la misma no sea necesariamente positiva. La CTI podría incrementar la desigualdad, tanto vertical (concentración de ingreso) como horizontal (de género, de raza, geográficas, etcétera). Los problemas asociados a la desigualdad son esencialmente sociales, y refieren a la desigual distribución de los recursos económicos, culturales y de poder, en ese sentido, requieren soluciones políticas y sociales, más que exclusivamente tecnológicas.

Lo anterior es un efecto o defecto que América Latina quizá se tendría que ir achicando, y esto se logra, en parte, midiendo su capacidad tecnológica y actuando en consecuencia.

Vemos pues que el estudio y el enfoque en la capacidad tecnológica trasciende más allá de los beneficios directos que se perciben en forma de crecimiento económico, sino que al ser por definición la capacidad de adoptar, adaptar y mejorar tecnologías, involucra elementos de la capacidad de absorción o aprendizaje que tienen que ver con educación y otros elementos contextuales que bajo esta investigación son analizados con proxies pero que sin duda tienen que ver con la calidad del entorno de las naciones y por otra parte tiene que ver con la capacidad innovativa de las naciones, es decir con la habilidad de generar efectos comercialmente plausibles y generar beneficios socio-tecnológicos. Esto último es lo que sin duda genera externalidades que econométricamente es un gran desafío medir y evaluar.

Es por ello pertinente no sólo ensanchar las investigaciones en este campo. Si no, sobre todo lograr tener eco dentro de las agendas ciudadanas de innovación, en la cual, por cierto, Iberoamérica recientemente cuenta con la propia. Además, tal 
como aconseja Albornoz (2001) es aún pertinente contar con una estrategia orientada a generar capacidades comunes que aglutinen a los científicos y a los centros de I+D latinoamericanos, tanto en el nivel regional como el subregional, ya que sólo a través de una estrategia de este tipo se puede alcanzar una dimensión equivalente a la de un país industrializado de tamaño medio.

Adicionalmente, Mongue y Tacsir (2013), encuentran que es necesario seguir investigando y establecer un mejor sistema de recopilación de datos en la región con el fin de evaluar las politicas de innovación de múltiples niveles de gobierno. En ese sentido, se debería echar mano de aquellas aplicaciones que estan haciendo que las estadisticas y la información cobren sentido de una manera mucho más amigable a través de la vizualización de datos y de la transparencia de infomación. ${ }^{26}$

Por otra parte, es necesario considerar que, como dice el dicho, no puedes enseñar trucos nuevos a un perro viejo. En promedio, los individuos y las naciones más jóvenes tienen mayor capacidad de (y están más abiertos a) aprender. Consecuentemente, lo que separa a los países desarrollados de los que están en vías de desarrollo es más bien una brecha en cuanto a conocimiento más que en cuanto a recursos (Stiglitz y Greenwald, 2014). Sin embargo, en el contexto latinoamericano, a pesar de que la pirámide generacional aún no se encuentre invertida como en ciertos casos europeos, de nada servirá si la juventud que habita dicho contexto no se encuentra apoyada por una infraestructura tecnológica, legal, financiera, fiscal, jurídica, etcétera, que respalde su ímpetu de aprendizaje, de poco servirá la juventud de quienes primordialmente y con urgencia necesitan cubrir sus necesidades mucho más básicas para después pensar en aspectos de educación (por ejemplo). El clima pues de innovación se vuelve crucial para engendrar, acumular y consolidar capacidades tecnológicas en las empresas, naciones y regiones. En el entendido de que tal como anota Yunus (2008,) "no se acaba de entender el enorme potencial que tiene la tecnología para transformar la situación de las personas más pobres del mundo". Sin olvidar que en los lugares donde florece la innovación (territorios innovadores) por lo general glorifican el talento, más que el dinero (Oppenheimer, 2014). Y en ese sentido, la tendencia a excluir a los que no tienen ideas parece ser más fuerte que la tendencia a excluir a los que no tienen riquezas (Tedesco, 2010).

Stiglitz (2012), advierte que el invertir más en nuestra sociedad (educación, tecnología e infraestructura) y proporcionar más seguridad a los ciudadanos generará una economía más eficiente y dinámica, más coherente con lo que decimos ser, ofreciendo más oportunidades a segmentos más amplios de la sociedad. Hacer

${ }^{26}$ Véase: GobApp (2014), Apps para el Desarrollo, América Latina, Creative Commons. 
caso a esta premisa, involucra, por una parte, reconocer las dinámicas heterogéneas que experimenta Latinoamérica y por otra, reconocer los beneficios de la capacidad tecnológica de los países sobre sus condiciones sociales.

Finalmente, escribiendo desde la autocrítica es por demás sabido que los outputs de la capacidad tecnológica son multivariados y pueden también ser manifestados en modelos de utilidad, invenciones de código abierto, la balanza tecnológica de pagos, el secreto industrial, innovaciones logradas (identificadas mediante encuestas). Sin embargo, la ventaja ampliamente reconocida de la utilización de patentes radica en la disponibilidad de datos regularmente obtenidos con series temporales "largas" y la comparabilidad internacional. Decimos largas entre comillas, porque la compilación estadística de este tipo de variables, es inclusive en estas fechas un reto pendiente; varios países latinoamericanos muestran huecos en sus series, desconociendo entonces aspectos tan elementales como patentes, investigadores y esfuerzos realizados en I+D. Lo anterior, es un obstáculo para emprender estudios más minuciosos y con mayor alcance muestral dentro de la región.

Consideramos pertinente reconocer que el régimen de propiedad intelectual apropiado para los países en vías de desarrollo y los mercados emergentes es totalmente diferente del adecuado para los países industrializados avanzados. En esta área, incluso más que en otras, las políticas universales son inapropiadas. Además de que se deberían considerar formas alternativas de diseñar un sistema de innovación, con un mayor énfasis en los premios y en el código abierto. Un buen sistema de patentes, por ejemplo, tiene que prestar más atención a la divulgación, a los problemas de bloqueo y al diseño de los mejores sistemas para impugnar patentes (Stiglitz y Greenwald, 2014).

Finalmente, se debe tener en cuenta que, si bien la apertura comercial es empíricamente relevante para la creación y acumulación de capacidades tecnológicas, dado que, de hecho, "los países en vías de desarrollo que han alcanzado más éxito lo han conseguido gracias al comercio a través de las exportaciones" (Stiglitz, 2006), es imperativo poner acento en que dicha apertura comercial debería ser adecuadamente gestionada para asegurar que los beneficios sean, pues, mayores a los costes.

Consideramos la región latinoamericana, como terreno fértil para realizar investigaciones focalizadas en ciencia, tecnología e innovación a sabiendas que éstas son proclives a servir como fuente de información a los tomadores de decisiones.

\section{REFERENCIAS BIBLIOGRÁFICAS}

Albornoz, Mario (2001), "Política Científica y Tecnológica, Una visión desde América Latina”, Iberoamericana de Ciencia, Tecnología, Sociedad e 
120 ECONOMÍA TeORÍA Y PRÁCTICA • Nueva Época, número 48, enero-junio 2018

Innovación, Madrid, OEI, [en línea], disponible en: http://www.oei.es/revistactsi/numero1/albornoz.htm

Amarante, Verónica; Galván, Marco y Mancero, Xavier (2016), Desigualdad en América Latina: una medición global, CEPAL, [en línea], disponible en: http://www.CEPAL.org/es/publicaciones/40024-desigualdad-america-latina-medicion-global

Aparicio, Javier y Marques, Javier (2005), Diagnóstico y especificación de modelos de panel en Stata 8, CIDE, [en línea], disponible en: http://webcache. googleusercontent.com/search?q=cache:_EmTPB-UK0wJ:investigadores.cide.edu/aparicio/data/ModelosPanelenStata.doc $+\&$ c$\mathrm{d}=1 \& \mathrm{hl}=\mathrm{es}-419 \& \mathrm{ct}=\mathrm{clnk} \& \mathrm{gl}=\mathrm{mx}$

Archibugi, Daniele (1998), "In Search of a Useful Measure of Technological Innovation (to Make Economists Happy without Discontenting Technologists)", Technological Forecasting and Social Change, 34, pp. 253-277.

Banco Mundial (2015), América Latina y el Caribe (paises en desarrollo solamente), [en línea], disponible en: http://datos.bancomundial.org/region/LAC

Banco Interamericano de Desarrollo (2014), ¿Cómo repensar el desarrollo productivo? América Latina y España, Fondo de Cultura Económica, [en línea], disponible en:https://publications.iadb.org/handle/11319/6634?locale-attribute $=$ es

Baumert, Thomas y Heijs, Joost (2013), Los determinantes de Capacidad innovadora regional: Una aproximación econométrica al caso español, Madrid, Instituto de Análisis Industrial y Financiero.

Borrastero, Carina (2012), "Notas sobre la cuestión del excedente en las teorías evolucionistas neoschumpeterianas", Papeles de trabajo, Revista electrónica del Instituto de Altos Estudios Sociales de la Universidad Nacional de General San Martín, pp. 100-126.

Bortagaray, Isabel y Gras, Natalia (2013), "Politicas de ciencia, tecnología e innovación para el desarrollo inclusivo: tendencias cambiantes en America del Sur", en Crespi, Gustavo y Dutrénit, Gabriela (2013), Politicas de ciencia, tecnología e innovación para el desarrollo: la experiencia latinoamericana, México, FCCYT y LALICS, pp. 263-300.

Botella, Carlos y Suarez, Ignacio (2012), Innovación para el desarrollo en America Latina: una aproximación desde la cooperación internacional, Fundación Carolina, [en línea], disponible en: http://www.fundacioncarolina. es/wp-content/uploads/2014/08/AI78.pdf 
Brunner, José (2011), Chile: informe sobre capacidades tecnológicas, Instituto de Economía Política, [en línea], disponible en: http://desarrollohumano.cl/ idh/download/capac_tecno.pdf

Brynjolfsson, Erik y McAfee, Andrew (2013), The Second Machine Age: Work, Progress, and Prosperity in a Time of Brilliant Technologies, W. W. Norton. CEPAL (2007), Indicadores de capacidades tecnológicas en América Latina, Serie Estudios y Perspectivas, México, Naciones Unidas, [en línea], disponible en: http://www.CEPAL.org/es/publicaciones/5014-indicadores-de-capacidades-tecnologicas-en-america-latina

(2015), Tv-Novosti, Organización Autónoma sin Fines de Lucro, [en línea], disponoble en: https://actualidad.rt.com/sociedad/164649-CEPAL-pais-pobreza-america-latina

Crespi, Gustavo (2014), BID Mejorando vidas. Invertir en ideas, [en línea], disponible en: http://blogs.iadb.org/Ideasquecuentan/2014/12/08/inver tir-en-ideas/

Crespi, Gustavo y Dutrénit, Gabriela (2013), Políticas de ciencia, tecnología e innovación para el dsarrollo: la experiencia latinoamericana, México, FCCYT Y LALICS.

Crespi, Gustavo y Maffioli, Alessandro (2013), "Diseño y evaluación de incentivos fiscales para la innovación empresarial en América Latina", en Crespi, Gustavo y Dutrénit, Gabriela, Politicas de ciencia, tecnologia e innovación para el desarrollo: la experiencia latinoamericana, México, FCCYT y LALICS, pp. 233-261.

Chinaprayoon, Chinawut (2007), Science, Tecnology and Innovation composite indicators for developing countries, Georgia Institute of Tecnology.

Diaconu, Mihaela (2011), "Technological Innovation: Concept, Process, Typology and Implications in the Economy", Theoretical and Applied Economics, 18 (10), pp. 127-144.

Dosi, Giovanni (2008), "La interpretación evolucionista de las dinámicas socioeconómicas", en Viale, R., Las nuevas economías. De la economía evolucionista a la economía cognitiva: Más allá de las fallas de la teoría neoclásica, México, pp.29-44.

Franco, Mercedes; Ramos, Lucrecia y Hernández, Yunieski (2016), Combinación de datos de corte transversal y de series temporales, Cuba, Universidad de Oriente.

Gans, Joshua y Hayes, Richard (2009), Assessing Australia's Innovative Capacity: 2009 Update, Australia, Melbourne, Business School and Intellectual Property Research Institute of Australia, the University of Melbourne. 
García-Ochoa Mayor; Mónica, Blázquez de la Hera; María. L. y López Sánchez, José I. (2012), Uso y aplicación de la técnica de análisis estadístico multivariante de cluster sobre la capacidad de innovación tecnológica en Latinoamérica y España, Innovar, 22, pp. 21-40.

GobApp (2014), Apps para el Desarrollo, América Latina, Creative Commons. Hernández, Gonzalo (2016), "La desigualdad es el problema en latinoamérica", [en línea], disponible en: http://revistafal.com/la-desigualdad-es-el-problema-en-latinoamerica/\#.Vy3rz6mdMWs.twitter. [Consultado el 1 de mayo de 2016].

$\mathrm{Hu}$, Mei y Mathews, John (2008), "China's national innovative capacity", Research Policy, pp. 1465-1479.

Labra, Romilio y Torrecillas, Cecilia (2014), Guía cero para datos de panel: Un enfoque práctico, Madrid, UAM-Accenture.

López del Paso, Rafael (2014), "Medición del grado de apertura de una economía", [en línea], disponible en: http:/www.extoikos.es/n14/pdf/18.pdf

Mayorga, Mauricio y Muñoz, Evelyn (2000), La técnica de datos de panel una guía para su uso e interpretación, Costa Rica, DIE.

Mongue, Ricardo y Tacsir, Ezequiel (2013), "Coordinación de la política de la IED”, en Crespi, G. y Dutrénit, G., Politicas de ciencia, tecnología e innovación para el desarrollo: la experiencia latinoamericana, México, FCCYT y LALICS, pp. 209-232.

Montero, Roberto (2011), Efectos fijos o aleatorios: test de especificación. Documentos de Trabajo en Economía Aplicada, España, Universidad de Granada, [en línea], disponible en: http://www.ugr.es/ montero/matematicas/especificacion.pdf

Morales, Eugenia; Ortiz, Carolina y Arias, Mayra (2012), "Factores determinantes de los procesos de innovación: una mirada a la situación en Latinoamérica”, Revista EAN, (72), pp. 148-163.

Navarro, Juan; Benavente, José y Crespi, Gustavo (2016), The New Imperative of Innovation: Policy Perspectives for Latin America and the Caribbean, New York, Inter-American Development Bank, [en línea], disponible en: https://publications.iadb.org/handle/11319/7417

OECD iLibrary (2015), Perspectivas económicas de América Latina 2016: Hacia una nueva asociación con china, Paris, OCDE 2.

Oppenheimer, Andrés (2014), Crear o morir: la esperanza de América Latina y las 5 claves de la innovación, México, Debate. 
Osorio, José (2009), "Recurso humano en México para la investigación educativa”, en Martínez, A.; López, P.; García, A. y Estrada, S., Innovación y competitividad en la sociedad del conocimiento, México, Plaza y Valdés, pp. 535-558.

Piñón, Francisco (2010), "Ciencia y tecnología en América Latina:una posibilidad para el desarrollo”, Globalización, Ciencia y Tecnología, pp. 29-39. Quiñones, Ainoa y Tezanos, Sergio (2011), “Ayuda oficial al desarrollo cientifico tecnológico: una evaluación macroeconómica de la distribución geografica y sectorial", Revista de Economía Mundial, pp. 151-177.

Ríos, Viridiana (2014), "Exportaciones de importaciones", Excelsior.

Romer, Paul (1990), "Endogenous Technological Change", Journal of Political Economy, 98, pp. 71-102.

Sachs, Jeffrey y Vial, Joaquín (2012), Competitividad y crecimiento económico en los países andinos y en América Latina, Cambrige, CAF.

Schumpeter, Joseph (2005), "Development”, Journal of Economic Literature, pp. 108-120.

SciDev (2015), Investigación y Desarrollo, [en línea], disponible en: http://invdes.com.mx/ciencia-mobil/8282-cuantos-cientificos-hay-que-tener

Stern Scott; Porter Michael y Furman Jeffrey (2000), The determinats of innovation capability, NBER Working Paper Series.

Stiglitz, Joseph (2012), The Price of Inequality: How Today's Divided society Endangers Our Future, W.W. Norton.

Stiglitz, Joseph y Greenwald, Bruce (2014), La creación de una sociedad del aprendizaje, México, Crítica.

Tedesco, Juan (2010), Educar en la sociedad del conocimiento, México, Fondo de Cultura Económica, [en línea], disponible en: https://socioeducacion. files.wordpress.com/2011/05/tedesco-carlos-educar-en-la-sociedad-del-conocimiento.pdf

Torres, Oscar (2007), Panel Data Analysis Fixes and Random Effects using Stata, USA, Princeton University, [en línea], disponible en: https://www.princeton.edu/ otorres/Panel101.pdf

Ulku, Hulka (2004), R\&D, Innovation, and Economic Growth: An empirical analysis, IMF Working Paper, pp. 4-28.

Yunus, Muhammad (2008), Un mundo sin pobreza, España, Paidós. 
